\title{
Statistics of the Electrosensory Input in the Freely Swimming Weakly Electric Fish Apteronotus leptorhynchus
}

\author{
Haleh Fotowat, ${ }^{1}$ Reid R. Harrison, ${ }^{2}$ and Rüdiger Krahe ${ }^{1}$ \\ 'Department of Biology, McGill University, Montréal, Québec H3A 1B1, Canada, and 'Intan Technologies, LLC, Los Angeles, California 90045
}

\begin{abstract}
The neural computations underlying sensory-guided behaviors can best be understood in view of the sensory stimuli to be processed under natural conditions. This input is often actively shaped by the movements of the animal and its sensory receptors. Little is known about natural sensory scene statistics taking into account the concomitant movement of sensory receptors in freely moving animals. South American weakly electric fish use a self-generated quasi-sinusoidal electric field for electrolocation and electrocommunication. Thousands of cutaneous electroreceptors detect changes in the transdermal potential (TDP) as the fish interact with conspecifics and the environment. Despite substantial knowledge about the circuitry and physiology of the electrosensory system, the statistical properties of the electrosensory input evoked by natural swimming movements have never been measured directly. Using underwater wireless telemetry, we recorded the TDP of Apteronotus leptorhynchus as they swam freely by themselves and during interaction with a conspecific. Swimming movements caused low-frequency TDP amplitude modulations (AMs). Interacting with a conspecific caused additional AMs around the difference frequency of their electric fields, with the amplitude of the AMs (envelope) varying at low frequencies due to mutual movements. Both AMs and envelopes showed a power-law relationship with frequency, indicating spectral scale invariance. Combining a computational model of the electric field with video tracking of movements, we show that specific swimming patterns cause characteristic spatiotemporal sensory input correlations that contain information that may be used by the brain to guide behavior.
\end{abstract}

\section{Introduction}

Characterizing the statistics of natural sensory stimuli is an important step for discovering how the nervous system extracts behaviorally relevant information from the world (Barlow, 1961; Simoncelli and Olshausen, 2001; Kurtz and Egelhaaf, 2003; Geisler, 2008). These statistics should ideally be measured in freely moving animals taking into account the concurrent, often actively controlled, movements of their sensory receptors (Schroeder et al., 2010; Egelhaaf et al., 2012). However, tracking the movement of receptor arrays and measuring their physical input in freely moving animals is often challenging. In the visual system, for example, the statistics of sensory input has been mostly studied using videos taken from natural scenes or by headmounted video cameras (Dong and Atick, 1995; Billock et al., 2001; Kayser et al., 2003). Nevertheless, the effect of eye movements on these statistics remains poorly understood.

The South American weakly electric fish Apteronotus leptorhynchus is an excellent model system for characterizing the sta-

\footnotetext{
Received March 6, 2013; revised July 9, 2013; accepted July 12, 2013.

Author contributions: H.F. and R.K. designed research; H.F. performed research; H.F. and R.R.H. contributed unpublished reagents/analytic tools; H.F. analyzed data; H.F. wrote the paper.

This work was supported by the Fonds Quebecois de Recherche sur la Nature et les Technologies and the Natural Sciences and Engineering Research Council of Canada (grants to R.K. and a postdoctoral fellowship to H.F.). The initial support for this project was provided by the Grass Foundation. We thank Dr. John Maunsell for lending us the two video cameras used for video tracking, Drs. Mansour Jamzad, Mark Nelson, and Jonathan Webb for helpful insights and suggestions, and Dr. Fabrizio Gabbiani for valuable comments on the manuscript.

The authors declare no competing financial interests.

Correspondence should be addressed to Haleh Fotowat, Department of Biology, 1205 Docteur Penfield, Room W3-23A, McGill University, Montréal, Québec H3A 1B1, Canada. E-mail: haleh.fotowat@mcgill.ca.

DOI:10.1523/JNEUROSCI.0998-13.2013

Copyright $\odot 2013$ the authors $\quad 0270-6474 / 13 / 3313758-15 \$ 15.00 / 0$
}

tistics of sensory input caused by an animal's self-generated movements, which are often actively controlled to acquire information about the environment (Stamper et al., 2012; Hofmann et al., 2013). These fish generate a quasi-sinusoidal electric field through an electric organ discharge (EOD). Thousands of cutaneous electroreceptors that are mostly sensitive to amplitude modulations (AMs) of the oscillating transdermal potential (TDP) sense changes in the electric field caused by body movements, nearby objects, and the electric fields of conspecifics (Bastian, 1981; Carr et al., 1982; Bastian, 1995). As the electroreceptors are distributed on the skin, their physical input can be measured transdermally and their position can be determined by tracking the fish's body, an important experimental advantage.

Investigating the characteristics of the electrosensory input has played a pivotal role in unraveling neural mechanisms underlying behavior (Fortune, 2006). For example, the discovery of the jamming avoidance response, in which the fish changes its EOD frequency when exposed to the electric field of a conspecific with similar frequency, has led to the complete description of its underlying neural circuitry (Heiligenberg, 1991). This behavior serves to avoid conspecific-evoked low-frequency AMs that are in the same frequency range as those evoked by prey (MacIver et al., 2001) and could thereby interfere with prey detection (Heiligenberg, 1991).

Despite the wealth of knowledge about the anatomy and physiology of the electrosensory system (Bullock et al., 2005), the spatiotemporal characteristics of the electrosensory input evoked by natural swimming remain unknown. In fact, the electric field structure and its modulations due to body movements and conspecific EODs have only been measured in immobilized fish 
A

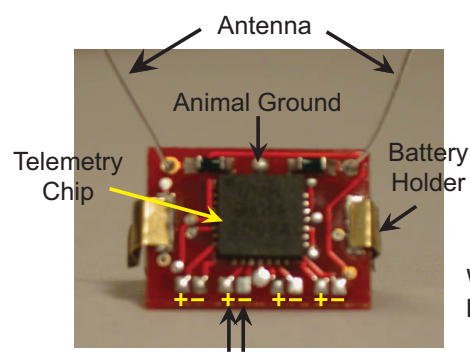

Input: Transdermal Potential
B

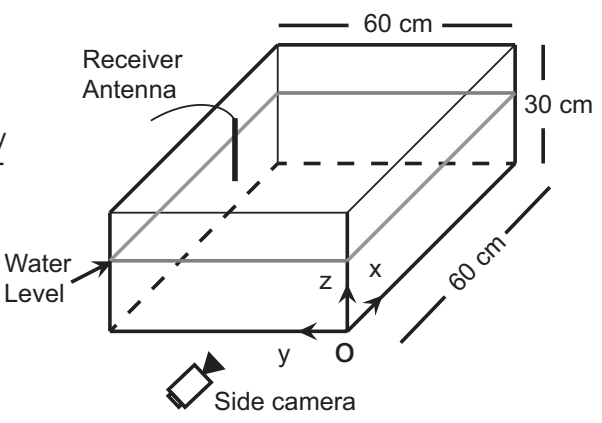

Figure 1. Wireless transmitter for recording the transdermal potential and the experimental setup. $\boldsymbol{A}$, Wireless transmitter consisted of a telemetry chip mounted on a $9 \times 13 \mathrm{~mm}^{2} \mathrm{PCB}$ board, which was powered by a pair of $1.5 \mathrm{~V}$ batteries. The whole board was made waterproof and sutured to the fish's skin like a backpack. The transdermal potential input to the board was amplified, filtered, digitized on board, and then transmitted digitally via the dipole antenna. $\boldsymbol{B}$, Fish's movements in the experimental tank were monitored using a pair of video cameras while acquiring its transdermal potential through a custom receiver with its antenna placed in the tank water. The cartesian coordinates of different points along the fish's body were defined relative to the location of the origin ( 0 ) and the direction of $x-y-z$ axes as indicated.

(Rasnow et al., 1993; Bastian, 1995; Assad et al., 1999; Chen et al., 2005; Yu et al., 2012).

We used a wireless transmitter to record the TDP of freely swimming weakly electric fish and characterized the dynamic range and spectral properties of the electrosensory input evoked by swimming and interactions with a conspecific. Combining a computational model of the electric organ with video tracking of the fish's body, we describe the spatiotemporal correlations across the sensory receptor array that arise from the organism's movements.

\section{Materials and Methods}

\section{Fish preparation for wireless telemetry}

We used the weakly electric fish A. leptorhynchus (the brown ghost knifefish) of either sex for the experiments. All animal care and surgical procedures were approved by the McGill University animal care committee. Fish were anesthetized in 100 ppm MS-222 (buffered with sodium bicarbonate to $\mathrm{pH} 7$ ) during transmitter positioning and electrode implantation. Three equal-length Teflon-coated silver wires $(75 \mu \mathrm{m}$ bare diameter, model 785500; A-M Systems) were used as recording, reference, and ground electrodes. The insulation was removed from the tip of the electrodes, at which point they were chlorided to achieve recording stability. The other ends of the electrodes were soldered to the transmitter board, which was then made waterproof and sutured to both sides of the fish's body slightly caudal to the gills and pectoral fins. The electrodes were forced to run parallel to each other along the fish's trunk by suturing them together to the skin at several points. A small hole was made in the fish's skin on the lateral mid-trunk using an insect pin and the recording electrode was slid through it for about $2 \mathrm{~cm}$ toward the tail. The reference and ground electrodes remained on the outside of the skin and were sutured to the skin caudal to the point of recording electrode insertion. After implanting the electrodes and positioning the transmitter backpack, the fish was placed in the experimental tank and allowed to recover from anesthesia for about $10 \mathrm{~min}$, by which time it had resumed normal swimming.

\section{Wireless transmitter}

We used a miniature wireless telemetry system (Harrison et al., 2011; Fig. $1 A$ ) to transmit the potential difference recorded across the fish's skin (the TDP) at a point on the mid-trunk of freely swimming fish. The transdermal potential was differentially amplified 1000 times, filtered in the range of $300-5200 \mathrm{~Hz}$, and sampled at $11.52 \mathrm{kHz}$ by a 9-bit analog-to-digital converter on board. It was then transmitted wirelessly at $920 \mathrm{MHz}$. The trans- mitter system's size is $13 \times 9 \mathrm{~mm}^{2}$ and it weighs $0.79 \mathrm{~g}$ including a pair of $1.5 \mathrm{~V}$ batteries (\#337; Energizer), which allows continuous signal transmission for $2 \mathrm{~h}$. A custom-built receiver captured the transmitted signal through a half-wavelength antenna and transferred it to the computer through a USB port, which was also used to power it. The tip of the receiver antenna was placed inside the tank water for the best signal reception (Fig. 1B). The wireless transmitter was made waterproof using liquid latex, beeswax, and vacuum grease after the electrodes were soldered to the input and ground ports.

\section{Experimental setup}

The experimental tank was $60 \times 60 \times 30 \mathrm{~cm}^{3}$ $(\mathrm{W} \times \mathrm{L} \times \mathrm{H})$, made of acrylic, and filled to about half-height with water (water depth varied between 15 and $20 \mathrm{~cm}$ across experimental sessions; Fig. 1B). Two video cameras (Guppy Pro, model \#F031C; Allied Vision Technologies) equipped with $\mathrm{f}=5 \mathrm{~mm}$ lenses (H0514MP2; Computar) were used to monitor the behavior of the fish from top and side views (Figs. $1 B, 2 A$ ) at a frame rate of $25 \mathrm{~Hz}$. The two cameras were synchronized with the TDP signal using TTL pulses that were generated by the acquisition computer and recorded through the receiver. Video and wireless recordings were acquired during multiple 20 -s-long periods (recording sequences). The distances of the cameras from the tank were set such that the field of view included the boundaries of the tank, resulting in a length-to-pixel ratio of $0.12 \mathrm{~cm}$ for the top-view camera.

\section{Quality of the wireless recordings}

The recordings were stable except occasionally when the receiver failed to receive any signal. These instances were clearly discernible visually as large amplitude noise in the received signal. The average (SD) duration of these episodes was $0.4 \mathrm{~s}(0.3 \mathrm{~s})$, after which the signal returned back to normal. These noise events did not occur at any particular time within or across recording sequences or relate consistently to the fish movements. The segments of the recording, which corresponded to the loss of reception, were identified based on the error bits generated by the receiver system and were removed from the recording sequence. The recording segments before and after the artifact were then joined together directly before subsequent analysis. Out of a total of $600 \mathrm{~s}$ of recordings from 3 single fish and $500 \mathrm{~s}$ from 2 pairs of fish, 60.2 and $87.3 \mathrm{~s}$ were discarded, respectively, due to such errors; the mean $(\mathrm{SD})$ of the total error time within a 20-s-long recording sequence was $2 \mathrm{~s}$ (1.9 s) and $3.5 \mathrm{~s}(2.5 \mathrm{~s})$ for single and pairs of fish, respectively. The higher relative total error time for the pairs of fish was likely due to physical contacts, which may have resulted in touching of the transmitter antenna by the second fish. We believe that removing these episodes from recordings did not significantly affect our results because they comprised $<20 \%$ of the total recording time, with individual episodes lasting on average $0.4 \mathrm{~s}$.

\section{Analysis of the transdermal potential recordings}

Single fish. All data analysis and modeling were carried out using custom MATLAB software (MathWorks). To extract the AMs of the EOD carrier signal, the TDP was first band-pass filtered to remove the EOD harmonics and occasional $60 \mathrm{~Hz}$ noise contamination. The filter's pass band was centered on the $f_{\mathrm{EOD}}$ and its bandwidth was equal to $800 \mathrm{~Hz}$. The time course of the TDP could then be simplified as follows:

$$
\mathrm{TDP}=V_{\mathrm{tdp}}(t) \times \cos \left(2 \pi f_{\mathrm{EOD}} t\right), V_{\mathrm{tdp}}(t) \geq 0
$$

Given that the temporal variation of $V_{\mathrm{tdp}}$ is much slower than $\cos (2 \pi$ $\left.f_{\mathrm{EOD}} t\right)$, it can be shown that $V_{\mathrm{tdp}}$ is equal to the amplitude of the analytic signal of the TDP, which can be calculated using the Hilbert transform (Zeimer et al., 1990): 


$$
\begin{aligned}
V_{\mathrm{tdp}}(t)=|\mathrm{TDP}+j \times \mathscr{H}(\mathrm{TDP})| & =\mid V_{\mathrm{tdp}}(t) \times \cos \left(2 \pi f_{\mathrm{EOD}} t\right)+j \\
& \times V_{\mathrm{tdp}}(t) \times \sin \left(2 \pi f_{\mathrm{EOD}} t\right) \mid .
\end{aligned}
$$

where $\mathscr{H}$ is the Hilbert transform, and $\mathrm{j}=\sqrt{-1}$. Low-frequency amplitude modulations caused by body movements were characterized using $V_{\mathrm{tdp}}(t)$ low-pass filtered at $50 \mathrm{~Hz}$. The power spectrum of the $V_{\mathrm{tdp}}$ for each recording sequence of each fish was normalized to the maximum power across all recording sequences for that fish. Only recording sequences that were $12 \mathrm{~s}$ or longer (after removing the segments with reception error) were used for calculating the power spectrum (with a total of 24 recording sequences in three fish). This restriction satisfied an equal FFT length for all sequences, which was chosen as the next power 2 from the length of the recording sequence and resulted in the FFT bin size of $0.044 \mathrm{~Hz}$. Given the maximum sequence length of $20 \mathrm{~s}$, the minimum resolvable frequency was $0.05 \mathrm{~Hz}$. The power-frequency plots are shown in logarithmic scale. The linear fit on the log-log plot was calculated using the least-squares method over frequencies smaller than $20 \mathrm{~Hz}$, where the low-pass filter had a flat magnitude response.

The percent change in TDP amplitude relative to the mean was calculated as follows:

$$
\Delta V_{\mathrm{tdp}}(t)=100 \times \frac{V_{\mathrm{tdp}}(t)-\bar{V}_{\mathrm{tdp}}}{\bar{V}_{\mathrm{tdp}}},
$$

where the $\bar{V}_{\text {tdp }}$ was calculated for each fish as the mean $V_{\text {tdp }}$ across all single-swimming recording sequences. The histogram for $\Delta V_{\text {tdp }}$ was estimated in bins of $1 \%$ width for both single and pairs of fish.

Pairs of fish. Two pairs of fish were studied in these experiments. The raw recordings were initially band-pass filtered with the filter's upper and lower limits extending $400 \mathrm{~Hz}$ above and below the larger and smaller $f_{\mathrm{EOD}} \mathrm{S}$ of the pair of fish, respectively. A Hilbert transform was then applied to the band-pass filtered recording and the resulting waveform was low-pass filtered at $400 \mathrm{~Hz}$ to estimate the AMs caused by body movements and the presence of the second fish. The AM signal showed significant power in the low-frequency range and around the frequency difference of the two fish (beat frequency, $\Delta f=70$ and $100 \mathrm{~Hz}$ for the pairs tested, with the transmitter fish having a higher and lower frequency than the other fish, respectively). When a conspecific came in close proximity of a fish, its electric field was added linearly to that of the fish's own at all points in space. The TDP at a point on one fish's skin (e.g., fish 1) can be simplified as follows:

$$
\mathrm{TDP}=V_{1}(t) \times \cos \left(2 \pi f_{\mathrm{EOD} 1} t\right)+V_{2}(t) \times \cos \left(2 \pi f_{\mathrm{EOD} 2} t\right) .
$$

where $f_{\mathrm{EOD} 1}$ and $f_{\mathrm{EOD} 2}$ are the EOD frequencies of each fish. $V_{1}(t)$ represents amplitude modulations caused by fish's own movement, and $V_{2}(t)$ represents the strength of the signal of fish 2 on the skin of fish 1 at the point of interest. Similar to equation 2, assuming that $V_{1}$ and $V_{2}$ vary much slower than their associated cosine terms, the amplitude of the TDP can be estimated using its analytic function:

$$
\begin{aligned}
V_{\mathrm{tdp}}(t)= & \mid V_{1} \times \cos \left(2 \pi f_{\mathrm{EOD} 1} t\right)+V_{2} \times \cos \left(2 \pi f_{\mathrm{EOD} 2} t\right)+j \\
& \times\left(V_{1} \times \sin \left(2 \pi f_{\mathrm{EOD} 1} t\right)+V_{2} \times \sin \left(2 \pi f_{\mathrm{EOD} 2} t\right)\right) \mid
\end{aligned}
$$

By trigonometry it can be shown that:

$$
V_{\mathrm{tdp}}(t)=\sqrt{V_{1}(t)^{2}+V_{2}(t)^{2}+2 \times V_{1}(t) V_{2}(t) \cos (2 \pi \Delta f t)},
$$

where $\Delta f$ is the beat frequency. Therefore, $V_{\text {tdp }}$ has power both at low frequencies and around the beat frequency, the spectral distribution of which is a function of the spectral distributions of $V_{1}$ and $V_{2}$. The amplitude modulations around the beat frequency (envelope) were extracted by first high-pass filtering the $V_{\mathrm{tdp}}$ above $50 \mathrm{~Hz}$ and then applying a Hilbert transform to the resulting waveform. The power spectrum of low-frequency $V_{\text {tdp }}$ and the envelope for the recording sequences in each fish pair were normalized to their respective maxima across recording sequences in that pair. Only recording sequences $12 \mathrm{~s}$ or longer were used for the spectral analysis (four recording sequences per fish pair). Leastsquares linear regression was used to fit the log-log plot of low-frequency $\mathrm{AM}$ and envelope powers for frequencies between 0 and $20 \mathrm{~Hz}$.

\section{Analysis of the video recordings}

The two cameras were first calibrated using an object marked with points with known $x-y-z$ coordinates. The calibration object consisted of a pair of cardboards marked at regular intervals along rectangular grids. One cardboard was placed on the bottom of the tank ( $z=0$ plane) and the other one was held at various $x$ positions at a $45^{\circ}$ angle relative to the first, amounting to a total of 99 points with known $x-y-z$ values distributed throughout the volume of the tank. Pairs of points with pixel coordinates $\left[u_{\mathrm{i}}, v_{\mathrm{i}}\right]$ and $\left[u_{\mathrm{i}}^{\prime}, v_{\mathrm{i}}^{\prime}\right]$ corresponding to a given point $\left[x_{\mathrm{i}}, y_{\mathrm{i}}, z_{\mathrm{i}}\right]$ were then manually identified in the pairs of images taken by the cameras ( $i=1: 99)$. A set of calibration coefficients was then calculated based on the direct linear transform (DLT) method with DLT tracking software (Hedrick, 2008 ) and used to calculate the $[x, y, z]$ values in centimeters of any points of interest in the video recordings. The DLT software was also used to track in 3D the position of the fish's head $(h)$, tip of its tail $(t)$, the wireless transmitter $(r)$, the approximate position of the electrode $(s)$, and a point in between the tip of its tail and the approximate electrode position $(m)$ (Fig. 2A, green crosses)

We used the MATLAB image processing toolbox to fit a polynomial to the fish's backbone and profile for each video frame captured from the top- and side-view cameras, respectively (Fig. $2 A$, red curves). The order of the polynomial was chosen between 3 and 5 for achieving minimal least-squares error. The polynomial fit to the backbone (Fig. $2 \mathrm{~A}$, top view) was initially constrained to pass through all the points identified in the previous step $(h, t, r, s, m)$. If a solution could not be found, the fitting condition was relaxed by reducing the number of points the curve had to go through, though always keeping points $h$ and $t$. The polynomial fit to the fish's profile was constrained to pass through $h, t$, and $m$, resulting in a curve that passed approximately through the middle of the trunk (Fig. $2 A$, side view).The fish's length in pixels $(L)$ was calculated as the median of the length of the curves fitted to its backbone across all video frames.

The speed of the fish relative to the nearby tank walls (i.e., those that were closer than $10 \mathrm{~cm}$ ) was calculated as the change in distance to those walls within successive video frames multiplied by the frame rate $(25 \mathrm{~Hz})$. For each recording sequence, we calculated the median of the speed relative to each tank wall. The average swimming speed relative to the nearby tank walls was then calculated as the average of the median speeds and used to divide recording sequences into two groups with average speeds larger or smaller than $0.25 \mathrm{~cm} / \mathrm{s}$ (Groups 1 and 2, respectively).

\section{Computational model of the electric field}

The fish's electric field was modeled by adapting the computational model of the electric organ proposed by Chen et al. (2005) for immobilized fish to the case of a freely swimming fish as follows. For each topview video frame, one negative electric charge ( pole) was assigned to the fish's tail (Fig. $2 B$, blue circle) and $L$-1-positive electric charges were distributed along the curve fitted to its backbone at equal distances (1 pole/pixel; Fig. $2 B$, red circles). The total positive electric charge of 20 $\mathrm{mV} / \mathrm{cm}$ was equally divided among the $L-1$ poles and the charge of the negative pole at the tail was set to $-20 \mathrm{mV} / \mathrm{cm}$. The charge spacing and amount were chosen to match the optimal values reported by Chen et al. (2005) for fish of comparable size. For each frame of the side-view camera, the corresponding pixel coordinates for the poles were calculated using the previously measured calibration coefficients with the constraint of being located on the curve fitted to the fish's profile. The positions of the poles in centimeters were then reconstructed in 3D for each frame based on their pixel values obtained from the top- and side-view cameras.

The effect of the non-conducting tank walls and the water surface (six non-conducting boundaries) was modeled using the method of image charges (Jackson, 1975; Chen et al., 2005). Briefly, due to the big conductivity difference between water and acrylic (or air), the normal component of the electric field at the boundary is effectively zero. One can thus model the effect of each boundary by replacing it with a mirror image of 
A

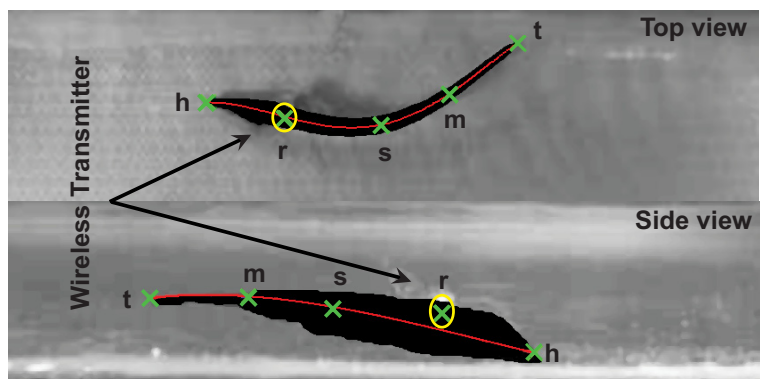

B

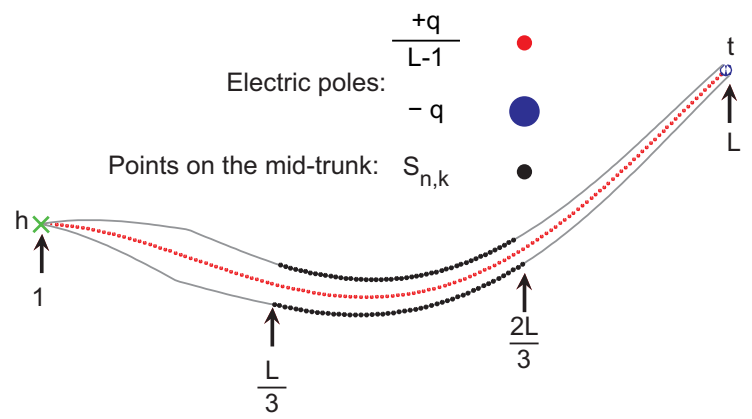

C

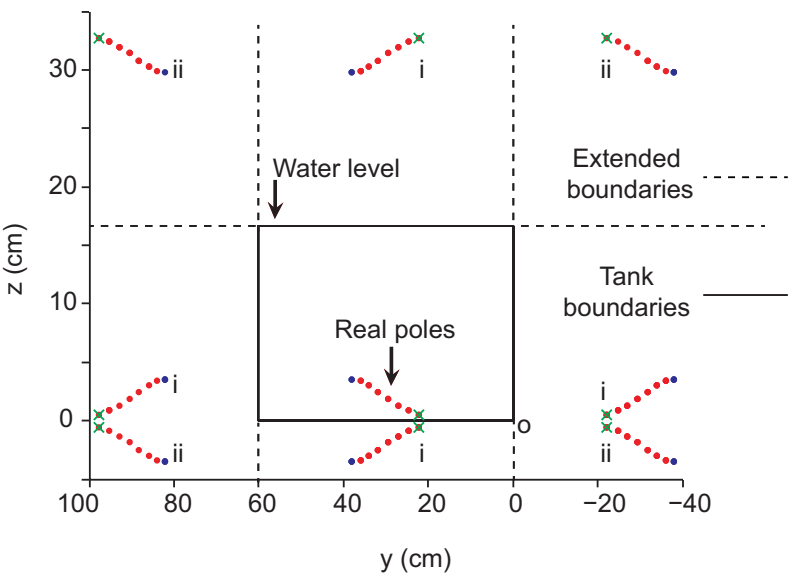

Figure 2. Tracking of the fish's midline and positioning of the model poles. A, Example of a pair of video frames captured by the top- and side-view cameras. The fish's body has been made darker for the purposes of this illustration. The position of the wireless transmitter $(r)$ and its approximate size relative to the fish are labeled with a green cross and a yellow circle, respectively. Other points of interest that were tracked across all video frames are marked with green crosses: $h, s, m$, and $t$. Polynomial fits to the backbone of the fish (top) and its profile (bottom) were calculated using the least-squares method and are plotted in red. $\boldsymbol{B}$, Positions of the positive and negative model electric poles for the same frame as shown in $A$ are marked with red and blue circles, respectively. The points on the mid-trunk $\left(S_{n, k}\right)$ for which the net electric field vector was calculated are marked with black circles. $C$, The position of the real electric poles corresponding to the fish's electric organ, their mirror images (i) calculated against the tank boundaries (solid lines), and the image of the image poles (ii) calculated against the extended tank boundaries (dashed lines) for the same frame as in $\boldsymbol{A}$ and $\boldsymbol{B}$. The effect of the tank boundaries on the electric field at any point in the space was taken as equivalent to the presence of the image fishes based on the method of image charges (see Materials and Methods). Red and blue circles correspond to positive and negative charges, respectively. Only a subset of poles and their images are shown as viewed in the $y-z$ plane for clarity. 0 , origin.

the real electric poles against that boundary. In the case of a rectangular tank to satisfy the boundary condition for zero normal component of the electric field, the charges should be symmetrical across all six surfaces. A subset of the image poles as viewed on the $y-z$ plane are shown in Figure $2 C$ (same frame as in Fig. $2 A, B$ ). In addition to the six images found from the real poles against the tank boundaries (Fig. $2 C, i$ ), images of the image poles should also be calculated across extended tank boundaries (Fig. $2 C$, ii), resulting in a total of 26 image fish.

We next calculated the net electric field for pairs of points $S_{\mathrm{nk}}(k=$ $[1,2])$ located on either side of each pole, $p_{n}$, in the mid-trunk area defined as the middle $30 \%$ of the fish's body length (Fig. $2 B$, black circles, point number $L / 3$ to point number $2 L / 3$ ). The $x$ and $y$ values of $S_{\mathrm{n}, \mathrm{k}}$ were chosen such that they were both at a distance $d_{\mathrm{n}}$ to $p_{\mathrm{n}}$ and that the line connecting them to $p_{\mathrm{n}}$ was locally orthogonal to the curve fitted to the backbone. The distance $d_{\mathrm{n}}$ was chosen such that it varied approximately in accordance with the natural variation in the width of the fish along the rostro-caudal axis (Fig. $2 B$, gray curve). The points $S_{\mathrm{n}, \mathrm{k}}$ therefore correspond to points on the skin of the model fish. The $z$ values of the points were set equal to the $z$ value of $p_{\mathrm{n}}$, for simplicity. For each video frame the net electric field vector $\left(\vec{E}^{\text {model }}\right)$ was calculated as the vector sum of the electric field vectors contributed by all real and image electric poles (Jackson, 1975) as follows:

$$
\begin{aligned}
\vec{E}^{\text {models }}\left(S_{\mathrm{n}, \mathrm{k}}\right)= & \sum_{j=1}^{m \times(L-1)} \frac{\frac{q}{L-1}}{\left|\vec{S}_{\mathrm{n}, \mathrm{k}}-\vec{S}_{\mathrm{P}+}^{j}\right|^{3}} \times\left(\vec{S}_{\mathrm{n}, \mathrm{k}}-\vec{S}_{\mathrm{P}+}^{j}\right) \\
& \quad-\sum_{j=1}^{m} \frac{q}{\left|\vec{S}_{\mathrm{n}, \mathrm{k}}-\vec{S}_{\mathrm{P}-}^{j}\right|^{3}} \times\left(\vec{S}_{\mathrm{n}, \mathrm{k}}-\vec{S}_{\mathrm{P}-}^{j}\right),
\end{aligned}
$$

where $\vec{E}^{\text {model }}\left(S_{\mathrm{n}, \mathrm{k}}\right)$ is the net electric field vector estimated by the model, $q$ is the total charge of $20 \mathrm{mV} / \mathrm{cm}, L$ is the total number of real poles, $m$ is total number of real and image fishes, $\vec{S}_{\mathrm{n}, \mathrm{k}}$ are the vector coordinates of the point at which the field is being calculated, $\vec{S}_{\mathrm{p}+}^{j}$ and $\vec{S}_{\mathrm{p}-}^{j}$ are the vector coordinates of the $j^{\text {th }}$ positive and negative pole, respectively.

Theoretically, at any point $S_{\mathrm{n}, \mathrm{k}}$ on the skin, the amplitude of the component of the electric field vector orthogonal to the skin, $E_{\text {ortho }}$, is related to the amplitude of the transdermal potential, $V_{\mathrm{tdp}}$, as follows:

$$
V_{\text {tdp }}\left(S_{\mathrm{n}, \mathrm{k}}\right)=\vec{E}\left(S_{\mathrm{n}, \mathrm{k}}\right) \cdot \vec{N}\left(S_{\mathrm{n}, \mathrm{k}}\right) \times \frac{\rho_{\text {skin }}}{\rho_{\text {water }}}=E_{\text {ortho }}\left(S_{\mathrm{n}, \mathrm{k}}\right) \times \frac{\rho_{\text {skin }}}{\rho_{\text {water }}},
$$

where $\vec{E}\left(S_{\mathrm{n}, \mathrm{k}}\right)$ is the electric field vector, $\vec{N}\left(S_{\mathrm{n}, \mathrm{k}}\right)$ is the unit vector orthogonal to the skin at point $S_{\mathrm{n}, \mathrm{k}}, \cdot$ is the vector dot product, $\rho_{\text {skin }}$ is the specific conductivity of the skin, and $\rho_{\text {water }}$ is the bulk resistivity of the water (Rasnow and Bower, 1996; Chen et al., 2005).

\section{Statistical analysis}

The variability of linear fit slopes was quantified by SE as described in Moore and McCabe (2006). Variability in data was otherwise quantified as SD. The Kruskal-Wallis test (KWT) was used to compare the medians of populations across different treatments. The level of statistical significance is denoted by $p_{\mathrm{KWT}}$. ANCOVA was used to compare the slopes of linear fits to the log-log plots of power spectra. The level of statistical significance for this analysis is denoted by $p_{\text {ANCOVA }}$.

\section{Results}

\section{Wireless recording of TDP in freely swimming fish}

Brown ghost knifefish equipped with a transdermal electrode and a wireless transmitter backpack started exploring the experimental tank as soon as they recovered from anesthesia. They showed the typical forward and backward swimming and fin movements, with a preference to swim close to the walls and the bottom of the tank (Fig. 3A). Swimming movements caused transient modulations in the amplitude of TDP, $V_{\mathrm{tdp}}$ (Fig. $3 B$, TDP: black curve, $V_{\text {tdp }}$ : blue curve; see also Eqs. 1 and 2 in the Materials and Methods). Tail bends toward (away from) a given point on the skin and an increase (decrease) in the distance of that point from ipsilateral non-conducting boundaries had been previously associated with increases (decreases) in the $V_{\mathrm{tdp}}$ at that point in immobilized fish (Chen et al., 2005). In freely swimming fish, however, in- 
A

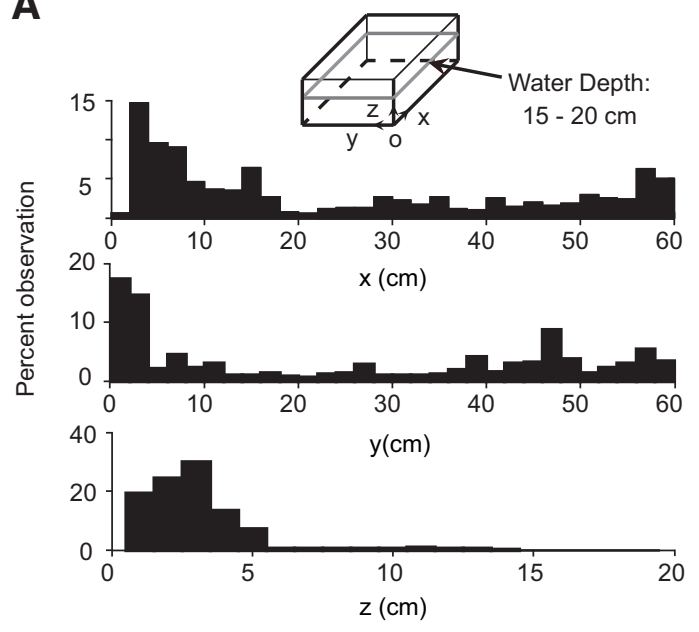

B

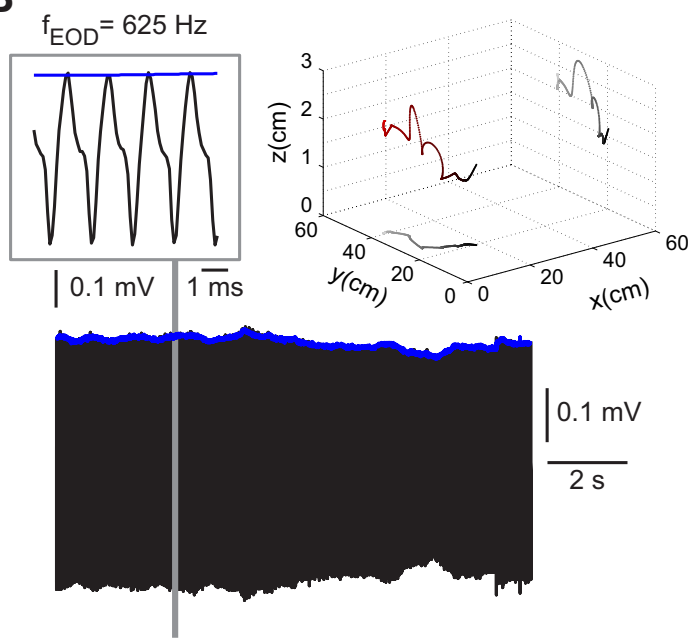

Figure 3. The distribution of the fish's positions in the experimental tank and an example wireless recording. $\boldsymbol{A}$, Probability distribution of the fish's positions in the experimental tank. Data were pooled across 3020 -s-long recording sequences obtained from three fish. We tracked the $x-y$-z position of the wireless transmitter across all frames in all fish (500 frames per recording sequence) and used it as an indicator of the fish's position. We chose the position of the transmitter for this purpose because it could be tracked automatically due to its large relative contrast. The fish tended to swim around the corners of the tank (large or small $x$ - $y$ values, top two) and near the bottom (small $z$ values, bottom). $\boldsymbol{B}$, Example recording of the TDP at a point on the mid-trunk (black curve) and its amplitude (blue curve). Inset: Top left, The gray area is shown on an expanded time scale to depict the quasi-sinusoidal electric organ discharge pattern ( $f_{\text {EOD }}=625 \mathrm{~Hz}$ ). Top right: The $x$ - $y$ - $z$ trajectory of the transmitter for this recording sequence (red points). The lighter shades of red correspond to the positions later in the sequence. The projections of this trajectory on $z=0$ and $x=$ 60 planes are shown in shades of gray, with lighter shades corresponding to the position later in the sequence. The water level was at $z=15 \mathrm{~cm}$ in this example, which is outside the range of the expanded scale shown here for the $z$-axis.

stances of increase or decrease in the $V_{\text {tdp }}$ were not clearly related to tail bends toward or away from the point of recording, because various body movements occurred at the same time. For example, the fish could be bending its tail to one side while also bending it in the dorsoventral plane. In addition, the distance to the boundaries of the tank could vary at the same time as a result of those body movements. To explain quantitatively the amplitude modulations observed in the wireless recordings, we tracked the details of the fish's body movements in 3D and then combined the video-tracking data with a computational model of the electric organ previously proposed for the case of immobilized fish (Chen et al., 2005).

\section{Estimation of the TDP amplitude based on the video recordings}

We tracked the shape of the fish's body in 3D across all video frames by fitting a polynomial to several key points along its midline (Fig. $2 A$, red curve). We modeled the electric organ as a negative electric charge (pole) located at the tip of the tail and a set of equally spaced positive poles along the midline, such that the sum of all positive charges was equal to that of the negative charge (Chen et al., 2005; see Materials and Methods). Using this model, we then calculated the electric field vector, $\vec{E}^{\text {model }}$, at evenly distributed points along the side of the fish's mid-trunk (see also Equation 7 in Materials and Methods and Fig. 2B).

We then calculated the orthogonal component of the electric field vector $\left(E_{\text {ortho }}^{\text {model }}\right)$ at each of those points. In theory, $V_{\text {tdp }}$ at any point on the skin should be proportional to the orthogonal component of the electric field vector at that point (Rasnow and Bower, 1996; see Equation 8 in Materials and Methods). We compared the Pearson correlation coefficient $\left(\rho_{\mathrm{p}}\right)$ between the $E_{\text {ortho }}^{\text {model }}$ calculated from the video recordings and that of $V_{\text {tdp }}$ obtained from the wireless recording down-sampled to the frame rate of the video recordings $(25 \mathrm{~Hz})$. The time course of the $E_{\text {ortho }}^{\text {model }}$ calculated at many points along the mid-trunk ipsilateral to the point of the implanted electrode was indeed highly correlated with the
$V_{\text {tdp }}$ measured from the wireless recordings (Fig. $4 A$, black circles). This correlation pattern did not exist at any point between the $E_{\text {ortho }}^{\text {model }}$ and random shuffles of the $V_{\text {tdp }}$ time series (Fig. $4 A$, green circles correspond to 10 random shuffles).

This result indicates that the model performs well in capturing the temporal dynamics in the $V_{\text {tdp }}$ caused by body movements and position changes relative to the tank boundaries. Because the exact position of the electrode could not be tracked from our video recordings, we chose the electrode position as the point in the mid-trunk region that showed the highest temporal correlation between its $E_{\text {ortho }}^{\text {model }}$ and the $V_{\text {tdp }}$ (Fig. $4 A$, blue vertical line: $\rho_{\text {p-max }}=0.72$ ). The point-by-point relation between $V_{\text {tdp }}$ and $E_{\text {ortho }}^{\text {model }}$ at $\rho_{\mathrm{p} \text {-max }}$ over the duration of the recording sequence could be well fitted using a least-squares linear regression (Fig. $4 B$, red line). The $V_{\mathrm{tdp}}^{\text {model }}$ calculated from $E_{\text {ortho }}^{\text {model }}$ using this linear relationship (Fig. $4 C$, red dots) captured the variations observed in the wirelessly acquired $V_{\text {tdp }}$ (Fig. $4 C$, blue dots) quite well (percent explained variance $=R^{2}=51 \%$ ). In this example, the points with larger estimation error (black asterisks) parallel the points that were off the linear fit (Fig. 4B, left) and correspond to instances where the fish made rolling movements (Fig. $4 B$, inset). Such errors are likely due to the error in the estimate of the electrode's $z$-position, which was set equal to that of the nearest electric pole in the model for simplicity (see Materials and Methods). Although this assumption is generally valid, it is violated during rolling movements. For the example shown, the rolling movements resulted in an increase in the distance of the electrode to the bottom of the tank, causing an increase in $V_{\mathrm{tdp}}$.

Table 1 shows the values for the correlation coefficient, slope, intercept, and $R^{2}$ for three recording sequences from the same fish. The corresponding $V_{\text {tdp }}$ calculated from the wireless recording and estimated from the model $\left(V_{\mathrm{tdp}}^{\text {model }}\right)$ for each sequence are shown in Figure $4 C$. The $E_{\text {ortho }}^{\text {model }}$ was linearly related to $V_{\mathrm{tdp}}$ as predicted, but with a non-zero intercept (bias). The bias in the $E_{\text {ortho }}^{\text {mod }}$, which was variable across recording sequences, could have been due to various estimation errors including inaccurate pre- 
A

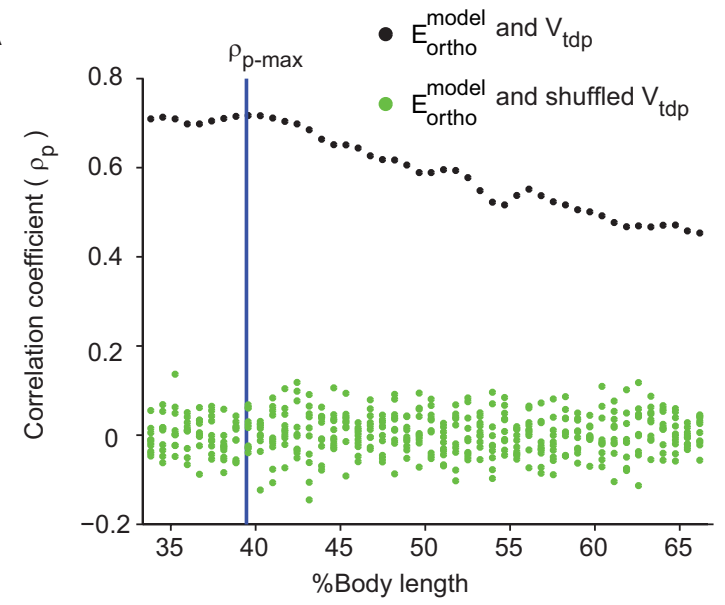

B

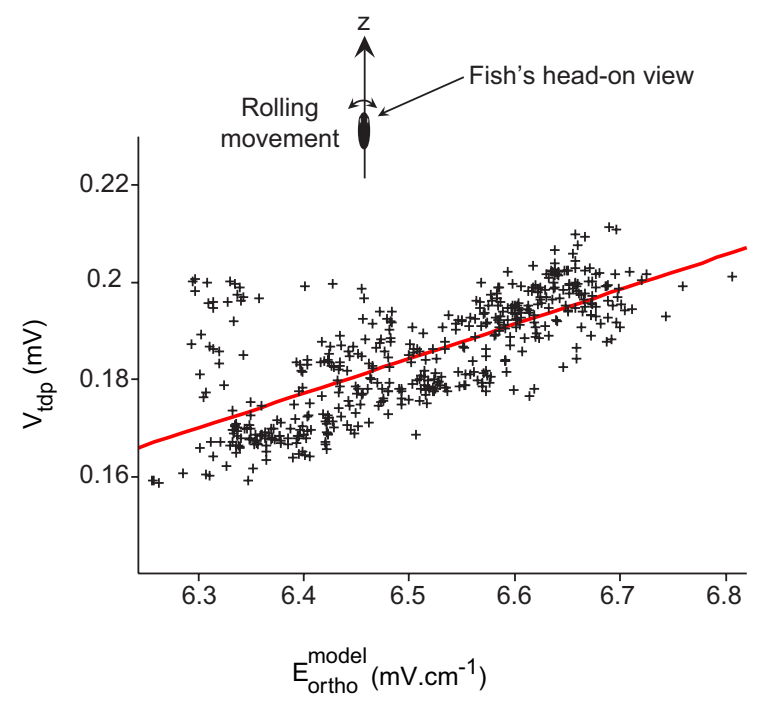

C

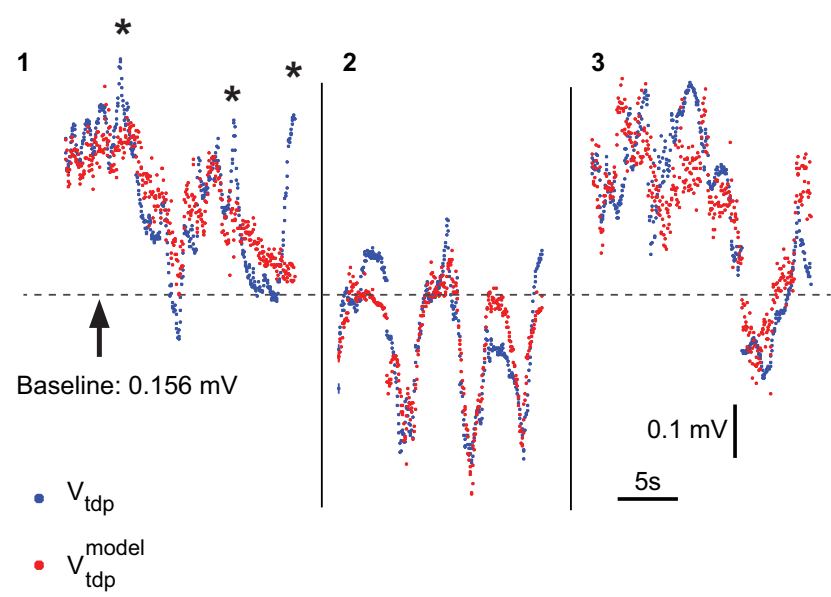

Figure 4. The estimation of transdermal potential based on the computational model of the electric field. A, At many points along the fish's mid-trunk (black circles), $V_{\text {tdp }}$ was highly correlated with $E_{\text {ortho }}^{\text {model }}$ calculated based on the video recordings and the computational model of the electric field (same recording sequence shown in Fig. $3 B$ ). The vertical blue line corresponds to the point with maximum correlation $\left(\rho_{\mathrm{p} \text {-max }}\right)$, which was taken as the approximate position of the implanted electrode. This correlation did not exist between random temporal shuffles of $V_{\text {tdp }}$ and the $E_{\text {ortho }}^{\text {model }}$ (green circles, 10 random shuffles). B, Relation between the $E_{\text {ortho }}^{\text {model }}$ and diction of the electrode position, limitations inherent in the linear model of the electric organ, and disregarding the effect of the fish's body on the shape of the electric field lines (Babineau et al., 2006). It should be noted that the slope and intercept of the linear fits were highly correlated $\left(\rho_{\mathrm{p}}=-0.98\right)$ indicating that the $E_{\text {ortho }}^{\text {model }}$ s estimation error is manifested in both parameters. In all recording sequences, however, we found an overall high degree of correlation between the $E_{\text {ortho }}^{\text {model }}$ and the wirelessly acquired $V_{\text {tdp }}$ at many points along the mid-trunk, ipsilateral to the position of the implanted electrode. Therefore, we are confident that the model is able to explain most of the variations in the transdermal potential of a single freely swimming fish measured through the wireless telemetry. We next used the computational model of the electric field to quantify the temporal correlation between the electrosensory input impinging on different points on the fish's mid-trunk.

\section{Movement-evoked spatiotemporal correlation patterns of electrosensory input}

Because the $V_{\mathrm{tdp}}$ at any point on the skin is a linear function of the orthogonal component of the electric field $\left(E_{\text {ortho }}^{\text {model }}\right)$ at that point (see Materials and Methods), we could quantify the movementspecific temporal correlations evoked in the $V_{\mathrm{tdp}}$ of any pairs of points on the skin by calculating the correlation coefficient between the time course of $E_{\text {ortho }}^{\text {model }}$ of those two points. We focused on three distinct swimming movements for this analysis: (1) side-to- side tail bends, (2) approaching a tank wall, and (3) body bends in the dorsoventral plane. As mentioned previously, the fish often made different body movements concurrently, making it difficult to isolate individual body movements.

We identified a recording sequence during which these movements could be approximately isolated and calculated the $E_{\text {ortho }}^{\text {model }}$ for points on either side of the mid-trunk (Fig. 2B, point number $L / 3$ to point number $2 L / 3$ on each side of the trunk; in this example, $L / 3,2 L / 3$, and $L$ were 47,95 , and 142 pixels, respectively). As an example, we show the $x-y$ - $z$ position and $E_{\text {ortho }}^{\text {model }}$ of a number of points located on the fish's mid-trunk (Fig. 5). Points $a$ and $b$ (Fig. $5 \mathrm{~A}$ inset, black and red larger circles, corresponding to points 78 and 88 of 142) are located on the same side of the trunk. Point $b^{\prime}$ is located directly across the midline from point $b$ on the contralateral side of the trunk (Fig. $5 \mathrm{~A}$, inset, larger purple circle). The time course of the $x-y-z$ movement of this point is very close to that of point $b$ and is not shown.

In the beginning of this recording sequence (Fig. $5 \mathrm{~A}, \mathrm{w} 1$ ), the fish was swimming far from the tank's side walls with all points along its midtrunk positioned $>10 \mathrm{~cm}$ away from the side walls, a distance at which large, non-conducting boundaries are thought to have minimal effect on the TDP (Fig. $6 \mathrm{~A}$ in Chen et al., 2005). During this time, the fish made occasional side-to-side tail bends and only slightly moved its trunk relative to the tank floor (Fig. $5 C, z$ ). We found that such body movements resulted in a positive correlation between the $E_{\text {ortho }}^{\text {model }}$ of points that were on the

$V_{\mathrm{tdp}}$ at $\rho_{\mathrm{p} \text {-max }}$ could be well fitted with a line (slope and intercept of the linear fit were $0.071 \mathrm{~cm}$ $\mathrm{SE}=0.003 \mathrm{~cm}$, and $-0.278 \mathrm{mV}, \mathrm{SE}=0.021 \mathrm{mV})$. The linear relationship is consistent with the theoretical relationship between the two (see Equation 8 in Materials and Methods). Inset shows the definition of a rolling movement, with the fish viewed head on. C, Wirelessly acquired $V_{\text {tdp }}$ (blue circles) and $V_{\text {tdp }}^{\text {model }}$ (red circles) as estimated from the linear fit in $\boldsymbol{B}$ in three different recording sequences from the same fish. The first recording sequence corresponds to Figure $3 B$. The parameters of the linear fit for these recording sequences (1-3) are presented in Table 1. Asterisks correspond to the time points around rolling movements. 
Table 1. $E_{\text {ortho }}^{\text {model }}-V_{\text {tdp }}$ linear fit parameters for three example recording sequences shown in Figure $4 C$

\begin{tabular}{llllll}
\hline $\begin{array}{l}\text { Recording } \\
\text { sequence }\end{array}$ & $\rho_{\mathrm{p}-\max }$ & Duration (s) & Slope (SE) (cm) & Intercept (SE) (mV) & $R^{2}(\%)$ \\
\hline 1 & 0.72 & 18.9 & $0.071(0.003)$ & $-0.278(0.021)$ & 51 \\
2 & 0.85 & 16.8 & $0.039(0.001)$ & $-0.084(0.007)$ & 74 \\
3 & 0.83 & 18.1 & $0.085(0.003)$ & $-0.457(0.021)$ & 68 \\
\hline
\end{tabular}

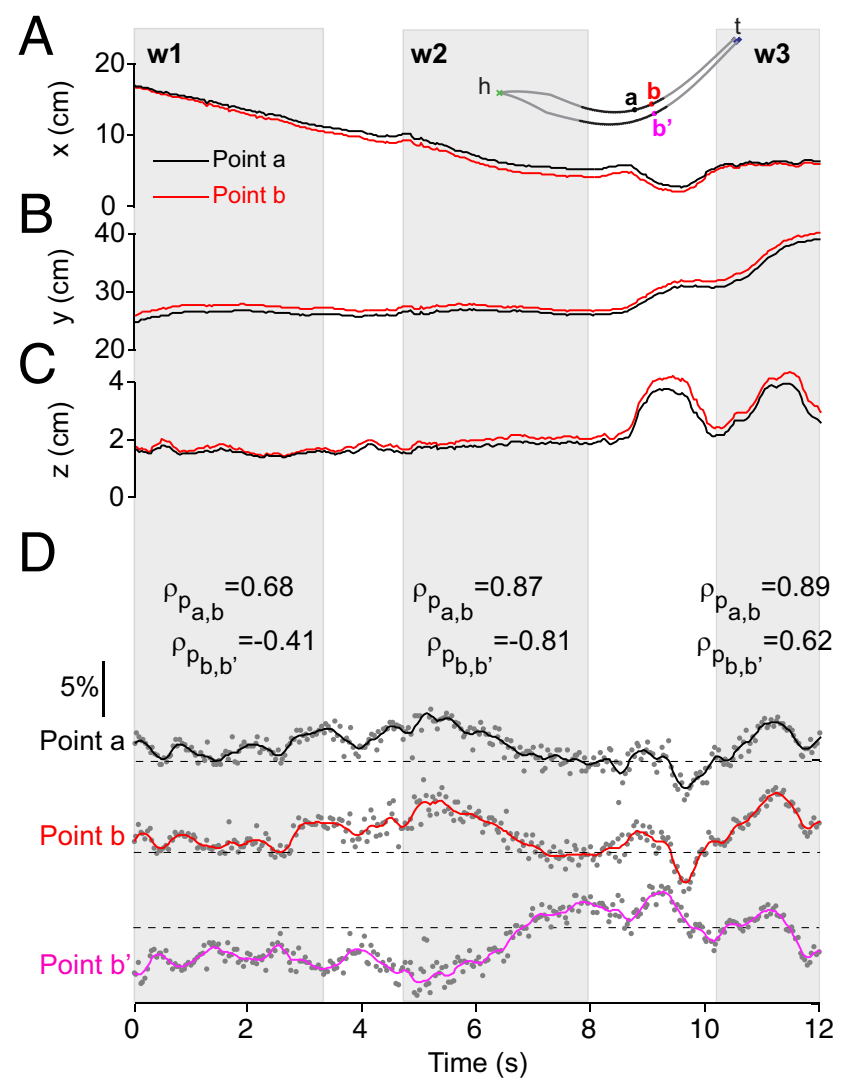

Figure 5. The position of example points on the fish's trunk and time course of the amplitude of the electric field orthogonal to the skin at those points during an example recording sequence. $\boldsymbol{A}-\boldsymbol{C}, x-y-z$ position of example points $a$ and $b$ (black and red curves, respectively) on the mid-trunk of the fish (inset). Shaded boxes mark the three time windows (w1-w3) that consisted of distinct swimming movements. During $w 1$, the fish was swimming far from the side walls of the tank $(x, y>10 \mathrm{~cm}, A, B)$ and made side-to-side body bends. During w2, the fish was approaching the tank wall corresponding to $x=0(x<10 \mathrm{~cm}, A)$ and, during w3, the fish was near the same wall and made body bends in the dorsoventral $(\boldsymbol{y}-\mathbf{z})$ axis. $\boldsymbol{D}$, Amplitude of the electric field vector orthogonal to the fish's skin $\left(E_{\text {ortho }}^{\text {model }}\right)$ normalized to its mean across the recording sequence is shown for the example points $a, b$, and b' (gray circles). The black, red, and purple curves are the five-point moving averages of the data for points $a, b$, and $b^{\prime}$, respectively. Vertical scale bar corresponds to percent change from the mean and $E_{\text {ortho }}^{\text {model }}$. $\rho_{\mathrm{P}_{2}}$ and $\rho_{\mathrm{P}_{\mathrm{a}, \mathrm{b}}}$ are the Pearson correlation coefficients calculated for the $E_{\text {ortho }}^{\text {model }}$ of points $\mathrm{a}$ and b, and points b and $b^{\prime}$, respectively. During $w 1$, the $E_{\text {ortho }}^{\text {model }}$ of points a and b were positively correlated with each other and negatively correlated with point $b^{\prime}$ located on the contralateral trunk. A similar, but stronger correlation pattern existed during w2. During w3, $E_{\text {ortho }}^{\text {model }}$ of all three points were positively correlated.

same side (e.g., points a and b, Fig. 5D, w1) and negative correlation between the points that were on opposite sides of the trunk (e.g., example points b and $\mathrm{b}^{\prime}$; Fig. 5D, w1).

The degree of temporal correlation in the $E_{\text {ortho }}^{\text {model }}$ of all pairs of points located on the same side (ipsilateral) and opposite sides (contralateral) of the trunk within this time window is presented in Figure 6, left $[(95-47+1=49) \times 49$ pairs of points, value of the Pearson correlation coefficient is represented by the color code]. The movements during time window w1 (Fig. 5) resulted in large-scale positive correlations between points on the same side of the body (Fig. 6A, top left) and negative correlations between points on opposite sides of the body (Fig. $6 A$, bottom left). To assess the extent to which the presence of tank boundaries could have contributed to these correlations, we removed them from the model by removing the image fishes (see Materials and Methods) and calculated the matrix of correlation coefficients again. As expected, because the fish was $>10 \mathrm{~cm}$ away from the tank side walls, removing them from the model did not affect the correlation pattern (Fig. $6 \mathrm{~A}$, right) and therefore these correlations were mainly due to the side-to-side tail bends.

During the second time window, the fish was swimming toward the tank wall located at $x=0$ (Fig. 5A, w2). As the fish approached the non-conducting boundary, there was an overall decrease in the amplitude of the $E_{\text {ortho }}^{\text {model }}$ for points facing the wall (e.g., points a and b, Fig. 5D, w2) and an overall increase for those on the opposite side (e.g., point b', Fig. 5D, w2). In fact, approaching the tank wall resulted in strong positive correlations between the sensory input impinging on all the points located on the same side and strong negative correlations between the points located on the opposite sides of the trunk (Fig. 6B, left). This correlation pattern was mainly imposed by movement relative to the tank walls because removing them from the model abolished it completely (Fig. 6B, right).

Body bends along the dorsoventral axis (up-down) are expected to affect the sensory input to points on either side of the trunk similarly and therefore cause positively correlated activity between the inputs to these points. Such correlations were indeed observed during the third time window (Fig. 5, w3), where the fish bent its body along the dorsoventral axis, causing an increase, peak, and decrease in the $z$ position of the points along the midtrunk (Fig. 5C, w3). As a result of this movement, the $E_{\text {ortho }}^{\text {model }}$ for points on either side of the midtrunk increased and then decreased (Fig. 5D, w3), giving rise to positive correlations, especially among the points closer to the tail (Fig. $6 \mathrm{C}$, left). Removing the tank boundaries had a relatively small effect on these correlations (Fig. 6C, right), indicating that they mainly resulted from the change in the structure of the electric field caused by the up-down body bends. The small effect of the tank walls on these correlations was expected because the distance to the tank's side walls was either large (Fig. 5B, w3) or relatively stable along the orthogonal axis (Fig. 5A, w3). It should be noted that although the distance of the points relative to the bottom surface of the tank (Fig. 5C, $z$, w3) was changing, this non-conducting boundary itself could not have an effect on the time course of the $E_{\text {ortho }}^{\text {model }}$ because the vector orthogonal to fish's trunk was set parallel to the bottom surface of the tank in our model.

The above analysis enabled us to isolate the major effects of body movements on spatiotemporal correlations of sensory input, the dynamics of which can inform the fish about multiple aspects of its interaction with its environment. For example, strong positive (negative) temporal correlation among the points on the same (opposite) side of the trunk, which translates into global in-phase/anti-phase synchronization in afferent activity, could be integrated by the next processing stages to signal the approach to a boundary. Similar analysis could be performed in future in the context of specific electrolocation tasks to shed light on the role of these correlations in guiding active sensation. 


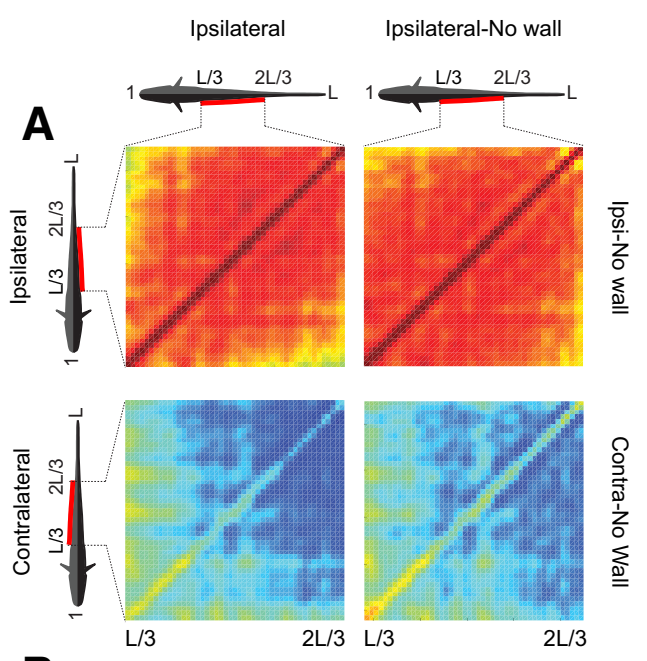

B
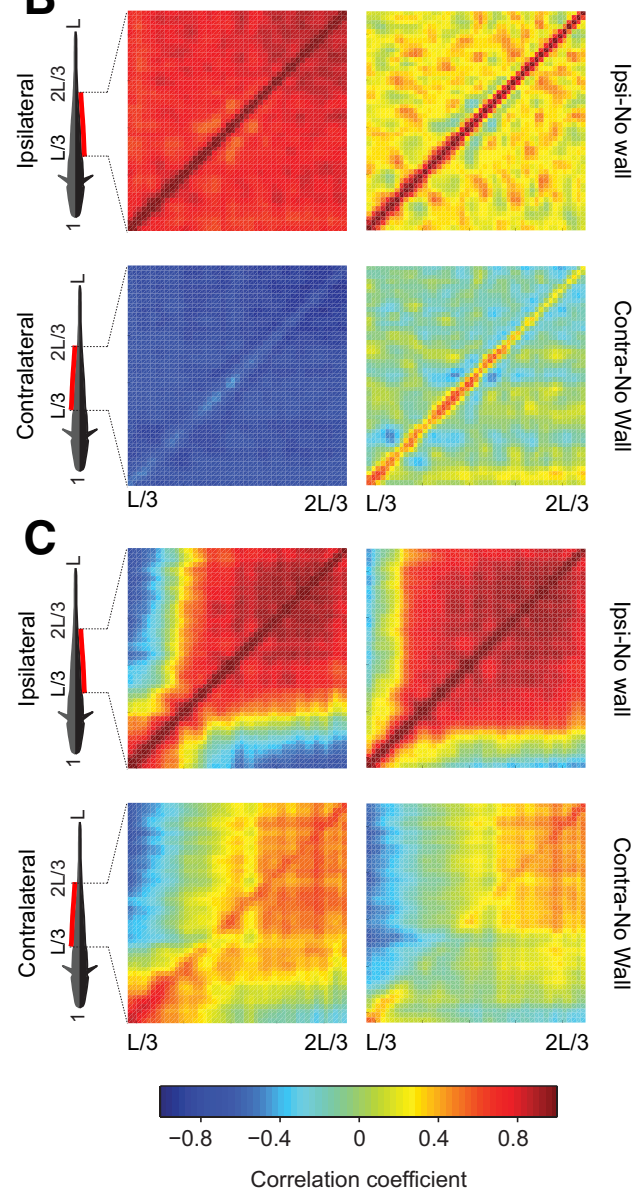

Figure 6. Spatiotemporal correlation patterns of the sensory input evoked by various swimming movements. $\boldsymbol{A}-\boldsymbol{C}$, Pearson correlation coefficients were calculated between the $E_{\text {ortho }}^{\text {model }}$ of pairs of points located on the same (ipsilateral) or opposite (contralateral) side of the body along the midtrunk in three time windows within a recording sequence (Fig. 5, w1-w3). Points located in the middle third of either side of the body (between $L / 3$ and $2 L / 3$, for a fish length of $L=142$ in this example, a total of 49 points on each side) were used for this calculation, resulting in a $49 \times 49$ correlation matrix. The value of the correlation coefficient is represented by the color code (bottom). The segment of the schematic fish highlighted in red corresponds to the side of the mid-trunk used for the calculation (gray or black corresponding to the right and left side, respectively). For $\boldsymbol{A}-\boldsymbol{C}$, the top left shows correlation coefficients among the points located ipsilaterally and the bottom left show the coefficients for points located contralaterally. The correlation matrix in the right top and bottom are calculated in the same manner after removing the tank walls from the model. $\boldsymbol{A}$, Side-to-side tail movements far away from the tank boundaries ( $d>10 \mathrm{~cm}$; Fig. $5, w 1)$ resulted in positive temporal correlation among the points

\section{Dynamics of the TDP amplitude evoked by swimming and interactions with a conspecific}

We used the wireless TDP recordings to quantify the range of the amplitude modulations $\left(\Delta V_{\mathrm{tdp}}\right)$ caused by natural swimming movements as the percent change from the mean $V_{\text {tdp }}\left(\bar{V}_{\text {tdp }}\right)$ estimated separately for each fish over all recording sequences (30 recording sequences, see Equation 3 in Materials and Methods). The means (SDs) of the $V_{\text {tdp }}$ for the three tested fish were as follows: fish 1: $0.157 \mathrm{mV}(0.013 \mathrm{mV})$ over $144 \mathrm{~s}$, fish 2: $0.105 \mathrm{mV}$ $(0.007 \mathrm{mV})$ over $156 \mathrm{~s}$, and fish 3: $0.276 \mathrm{mV}(0.018 \mathrm{mV})$ over $240 \mathrm{~s}$ of recording. We found that swimming movements within the non-conducting boundaries of the tank could cause AMs as large as $20 \%$ (Fig. 7A). The Gaussian fit to the distribution pooled across the three tested fish had an SD of 9\%, indicating that about $68 \%$ of the observed amplitude modulations were within $9 \%$ of the mean.

We next introduced a conspecific to the tank and continued measuring the TDP (two of the three fish tested individually were used for these experiments). The $V_{\mathrm{tdp}}$ measured at a point on the skin of one fish during its interaction with a conspecific is a function of the linear sum of the electric fields along the axis orthogonal to the fish's skin at that point. Because the electric organs of individual brown ghost knifefish discharge at individual-specific frequencies, the $V_{\mathrm{tdp}}$ will vary at the difference of their discharge frequencies (beat frequency, $\Delta f$ ), even in the absence of any body movements. During natural interactions with a conspecific, the amplitude of the $V_{\mathrm{tdp}}$ varies as a result of the fish's own body movements, its position change relative to the environment, and the conspecific (Fig. $7 B$, blue lines, $\Delta f=100 \mathrm{~Hz}$, see Equation 6 in Materials and Methods).

We found that interacting with a conspecific caused a much larger range of AMs compared with those caused by a single fish's swimming movements (Fig. $7 C, 10$ recording sequences from two pairs of fish amounting to a total of $165.36 \mathrm{~s}$ of recording). In this case, the same baseline value as used in the case of single swimming fish was used to calculate $\Delta V_{\text {tdp }}$. A Gaussian fit to the distribution had a SD of $19.7 \%$, with the tail of the distribution even extending up to $100 \%$. This is more than twice the SD of the Gaussian fit to the distribution of the AMs in the case of a single swimming fish (9.0\%). The amplitude of AMs around the beat frequency (Fig. $7 B$, envelope, red curve) changes as a function of the relative orientation of the two fish.

We found that at distances larger than $\sim 23 \mathrm{~cm}$ (distance calculated between the fish's midpoints), the envelope was always $<20 \%$ of the mean $V_{\text {tdp }}$ (Fig. $7 D$ ), a value that corresponds to the upper bound of AMs in the case of a single swimming fish (Fig. $7 A$ ). Conversely, at shorter distances, it was possible to get modulations of $<20 \%$ and up to $100 \%$ (Fig. $7 D$ ). Therefore, the distance between the two fish could not be a simple predictor of the AMs around the beat frequency and the relative orientation of fish bodies could affect the extent of these modulations signifi-

$\leftarrow$

on the same side of the trunk: ipsilateral (ipsi, top left) and negative correlation with the points located on the opposite side of the trunk: contralateral (contra, bottom left). Removing the tank walls in the model did not affect the correlation patterns (right). $\boldsymbol{B}$, Approaching a tank wall (Fig. 5, w2) resulted in very strong positive and negative correlation among the points located on the same and opposite sides of the trunk, respectively (left). Removing the tank walls in the model removes those correlations (right). C, Up-down tail movements (Fig. 5, w3) resulted in positive correlation among the points on the same side and the ones on opposite sides of the trunk (left). In this example, the modulations were not affected after removing the tank walls in the model because the orthogonal distance to the walls was relatively stable (right). 
A

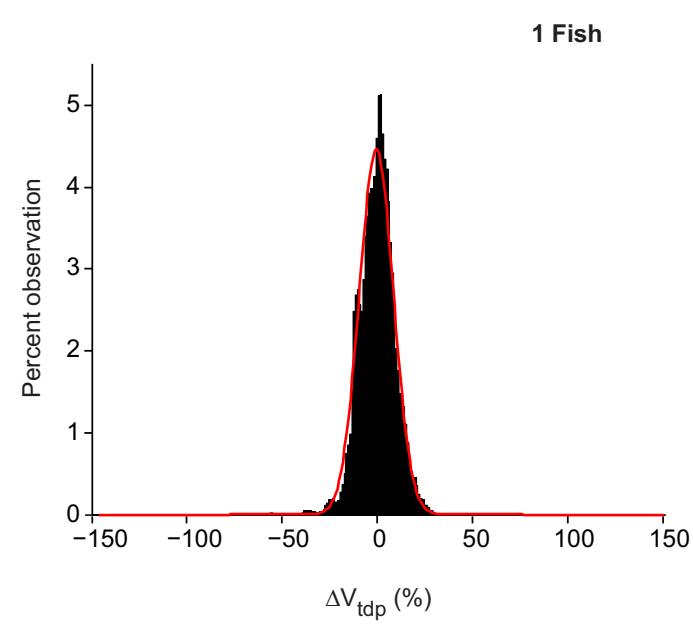

C

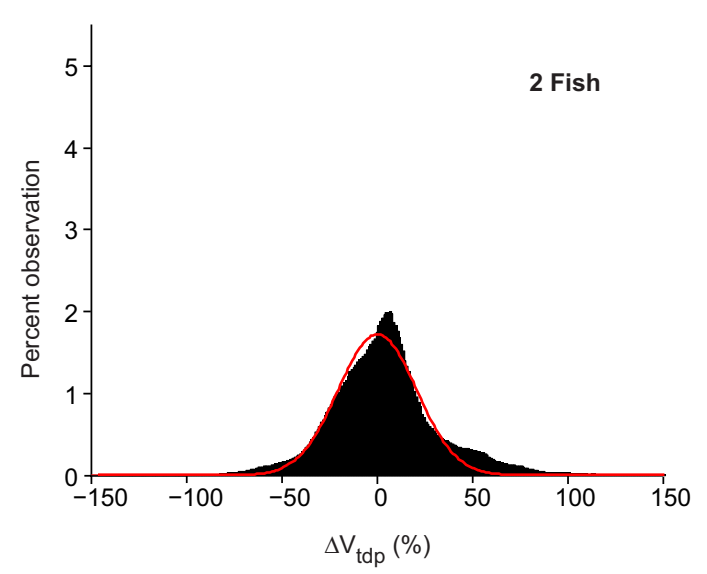

B
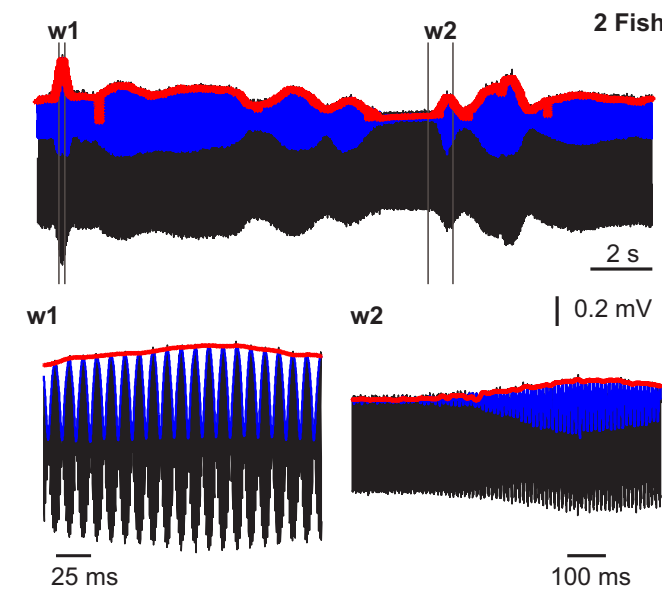

D

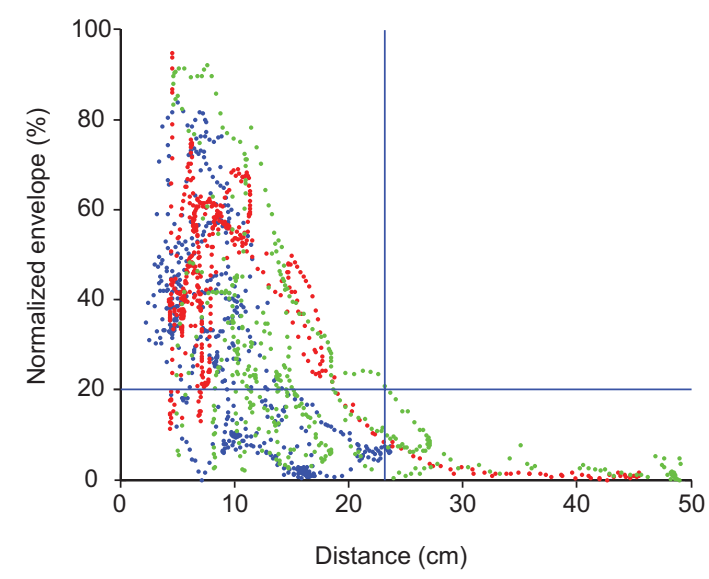

Figure 7. Range of transdermal potential amplitude modulations experienced by electric fish swimming alone and during interactions with a conspecific. $\boldsymbol{A}$, Range of observed amplitude modulations around the mean $V_{\text {tdp }}\left(\Delta V_{\text {tdp }}\right.$, see Equation 3 in Materials and Methods, data are from 30 recording sequences in three fish). The amplitude modulations caused by body movements and the resulting displacement relative to the non-conducting boundaries of the tank were generally $<20 \%$ of the mean. Red curve, Gaussian fit to the distribution (SD $=9 \%$ ). $\boldsymbol{B}$, During interaction with a conspecific, the net transdermal potential (black) is equal to the sum of the two EOD waveforms; therefore, its amplitude ( $V_{\text {tdp }}$, blue curve) oscillates at the difference frequency ( $100 \mathrm{~Hz}$ in this example). The amplitude of the $V_{\text {tdp }}$ at the beat frequency (envelope, red curve) varies as a function of relative body movements of the two fish. The recording is shown on an expanded time scale for two time windows (w1, w2) at the bottom. $C$, Range of modulations observed around the mean $V_{\text {tdp }}$ of one fish during interaction with a conspecific ( $\Delta V_{\text {tdp }}$, data are from 10 recording sequences in two pairs of fish). The AMs caused by interactions with a conspecific, body movements, and displacements relative to the non-conducting boundaries of the tank ranged up to $100 \%$ of the mean. Red curve, Gaussian fit to the distribution (SD = 19.7\%). $\boldsymbol{D}$, Examples of the relation between the inter-fish distance (distance between their centers) and the percentage of AMs around the beat frequency (envelope normalized to mean $V_{\text {tdp }}$ ) for three recording sequences obtained in the same pair of fish. Red, green, and blue dots show data points from three different sequences. The vertical blue line marks the maximum inter-fish distance at which modulations of $>20 \%$ (horizontal blue line) from baseline were observed. Although at distances longer than $23 \mathrm{~cm}$, the modulations were always smaller than $20 \%$, at shorter distances, the modulations could assume larger or smaller values.

cantly. To explain the variations observed in the case of two fish, the computational model described in the previous section must be developed further to account for the conspecific's electric field and its physical presence as an external conductive object, which is beyond the scope of the present study.

\section{Spectral characteristics of the AMs and envelopes}

The AMs caused by swimming movements within the nonconducting boundaries of the tank had power mainly at low frequencies, with the mean normalized power smaller than $10^{-4}$ for frequencies larger than $20 \mathrm{~Hz}$. The power-frequency relationship on the log-log scale could be generally fit well with a line for the full frequency range indicating a power-law relationship with frequency $\left(\right.$ power $=1 / \mathrm{f}^{\beta}$ ) with the power exponent $\beta=-1 \times$ slope of the linear fit (Fig. $8 A$, blue line; red curve is the median of the power spectra). The details of the structure of the power spectrum within this range, however, depended on the fish's swimming pattern in relation to the tank boundaries during a given recording sequence. We divided the recording sequences into two groups based on the fish's swimming speed relative to the tank boundaries. The first group consisted of recording sequences during which the fish was actively swimming with speeds larger than $0.25 \mathrm{~cm} / \mathrm{s}$ (Group 1, 15 recording sequences in 3 fish, average speed $=1.7 \mathrm{~cm} / \mathrm{s} ; \mathrm{SD}=1.2 \mathrm{~cm} / \mathrm{s}$ ) and the second group consisted of those during which the fish was either "hovering in place" or moving very slowly relative to the nearby tank walls and its swimming speed was smaller than $0.25 \mathrm{~cm} / \mathrm{s}$ (Group 2, 9 recording sequences in 3 fish, average speed $=0.15 \mathrm{~cm} / \mathrm{s} ; \mathrm{SD}=0.06 \mathrm{~cm} / \mathrm{s}$ ). Although for lower frequencies the power spectrum of the recording sequences 

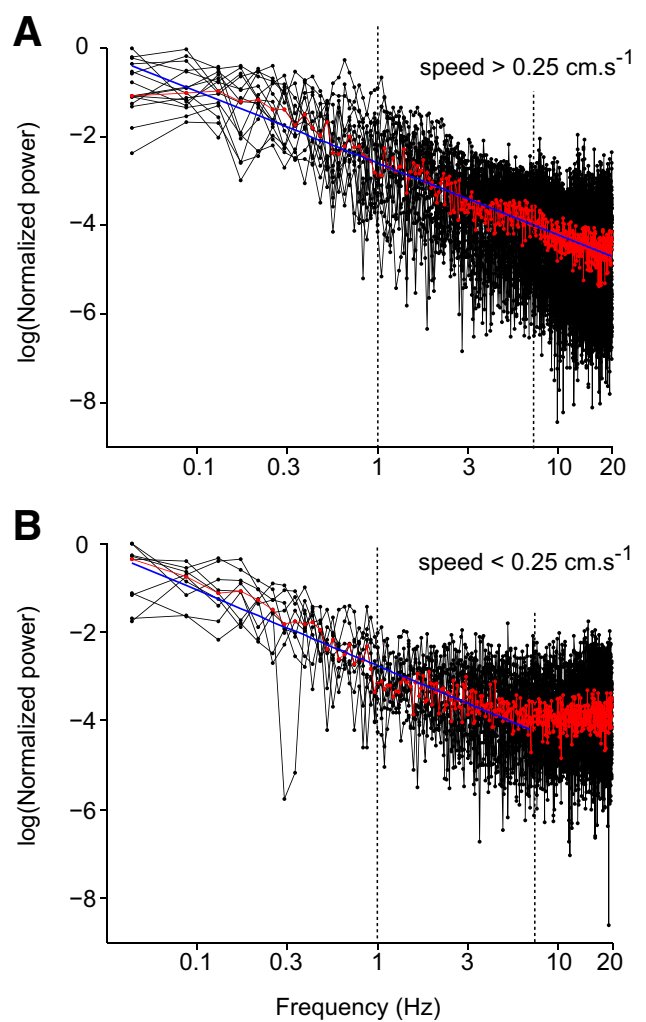

C

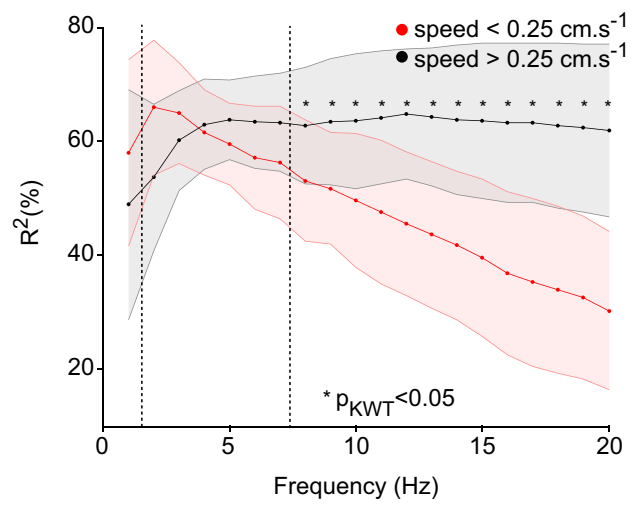

Figure 8. Spectral properties of the amplitude modulations in single swimming fish. $\boldsymbol{A}$, Normalized power spectrum of the $V_{\text {tdp }}$ plotted on a log-log scale (black) for all recording sequences $12 \mathrm{~s}$ or longer $(n=15)$ obtained from three single-swimming fish with relatively high activity level (average swimming speed $>0.25 \mathrm{~cm} / \mathrm{s}$ ) and their median (red). The blue line is the linear fit to the median (slope $=-1.62 ; \mathrm{SE}=0.029 ; R^{2}=87 \%$ ). Such a linear relationship indicates a power-law relationship with frequency with power exponent $\beta=1.62$ and spectral scale invariance. $\boldsymbol{B}$, Normalized power spectrum of the $V_{\text {tdp }}$ (black) for all recording sequences $(n=9)$ obtained from three single-swimming fish with low activity levels (average swimming speed $<0.25 \mathrm{~cm} / \mathrm{s}$ ) and their median (red). The blue line shows the linear fit to the median for frequencies smaller than $7 \mathrm{~Hz}$ (slope $=-1.57 ; \mathrm{SE}=0.005 ; R^{2}=85 \%$ ). C, Mean and $S D$ of the goodness of the linear fit $\left(R^{2}\right)$ for the recording sequences in which the swimming speed was larger (black) and smaller (red) than $0.25 \mathrm{~cm} / \mathrm{s}$. For upper-bound frequencies larger than $7 \mathrm{~Hz}$, the linear fit was significantly worse for the recording sequences with very slow swimming speeds $\left(p_{\mathrm{KWT}}<0.05\right)$.

from both groups could be well fit with a line in the log-log domain (Fig. $8 A, B$ ), the power spectrum of the AMs of the second group tended to resemble that of the white noise for larger frequencies (i.e., it flattened for larger frequencies and therefore could not be well fit with a line for the whole frequency range; Fig. $8 B$, red curve, median power spectrum).
Considering that the fish was only moving very slowly during these recording sequences, the low signal-to-noise-ratio for larger frequencies was expected. This result could be further quantified by calculating the goodness of the linear fit to the power spectra in different frequency bands with equal lowerbound frequency $(0.044 \mathrm{~Hz})$ and variable upper-bound frequencies $(1-20 \mathrm{~Hz}$ in $1 \mathrm{~Hz}$ steps; Fig. 8C). For upper bound frequencies smaller than $7 \mathrm{~Hz}$, the power spectrum of both groups could be equally well fit with a line $\left(R^{2}\right.$ values were not significantly different, $\left.p_{\mathrm{KWT}}>0.05\right)$, whereas for higher frequencies, the fit to the power spectra of the second group became significantly worse. The slopes of linear fits to the spectra of the recording sequences of the two groups for the upper-bound frequency of $7 \mathrm{~Hz}$ were not significantly different (Group 1: slope= $-1.81, \mathrm{SD}=0.26 ;$ Group $\left.2=-1.52, \mathrm{SD}=0.32 ; p_{\mathrm{KWT}}=0.07\right)$. These slopes were not significantly different from the slopes of the linear fits to the spectra of the recordings of the first group up to $20 \mathrm{~Hz}$ either $\left(p_{\mathrm{ANCOVA}}=0.15\right.$, mean slope Group 1 fit to the full range $=-1.72 ; \mathrm{SD}=0.39)$.

During interactions with a conspecific, $V_{\text {tdp }}$ showed variations at low frequencies and around the beat frequency (Fig. 9A). Both the spectral power of the low-frequency $(f<20 \mathrm{~Hz})$ component of the $V_{\mathrm{tdp}}$ (Fig. $9 \mathrm{~B}$ ) and that of the $V_{\mathrm{tdp}}$ around the beat frequency (Fig. 9C, envelope) decayed with increasing frequency, and the spectra could be equally well fit with a line on a log-log scale for the full frequency range $(f<20 \mathrm{~Hz}$; Fig. 9D).

For frequencies smaller than $1 \mathrm{~Hz}$, the goodness of the linear fit to the spectrum of the $V_{\mathrm{tdp}}$ of a single swimming fish (Fig. $8 \mathrm{C}$ ) and the spectrum of the low-frequency $V_{\text {tdp }}$ and the envelope during interaction with a conspecific (Fig. 9D) showed large variability across recording sequences. In the case of the single swimming fish, the $R^{2}$ could vary between $11 \%$ and $77 \%$. In the context of interacting with a conspecific, the $R^{2}$ values ranged between $2 \%$ and $57 \%$ for low-frequency $V_{\text {tdp }}$ and between $2 \%$ and $72 \%$ for the envelope. The variability in the power spectrum structure for frequencies smaller than $1 \mathrm{~Hz}$ was likely due to the difference in the low-frequency swimming patterns and/or movements relative to the non-conducting boundaries or the conspecific within the limited duration $(<20 \mathrm{~s})$ of individual recording sequences. It was, however, often difficult to relate specific patterns of swimming causally to the specific shape of the power spectrum due to the large diversity of the swimming movements that could occur within different recording sequences. Calculating the power spectrum over shorter segments of a recording sequence that contained specific swimming patterns could not help resolve low-frequency structure of the power spectrum either, because it resulted in an increase in the minimum resolvable frequency (1/duration of the recording). The problem becomes even more involved for the case of pairs of interacting fish. In the following section, we present a more detailed analysis of the power spectra of the $V_{\mathrm{tdp}}$ of single swimming fish using a few example recording sequences during which the fish's swimming patterns were relatively simple and could be classified in a rather straightforward manner.

In the course of a recording sequence in which the fish made slow approaching and receding movements relative to a tank boundary (Fig. 10AI, average swimming speed $=0.75 \mathrm{~cm} / \mathrm{s}$ ), the $V_{\text {tdp }}$ contained large low-frequency fluctuations (Fig. 10AII) and fluctuations at higher frequencies and the power spectrum could be very well fit with a line across all frequencies (Fig. 10AIII, red line, $\left.R^{2}=66 \%\right)$. The spectrum could be similarly well fit for frequencies smaller than $1 \mathrm{~Hz}\left(R^{2}=72 \%\right)$ and the slope of the linear fit for this frequency range (Fig. 10AIII, blue line, slope $=$ 

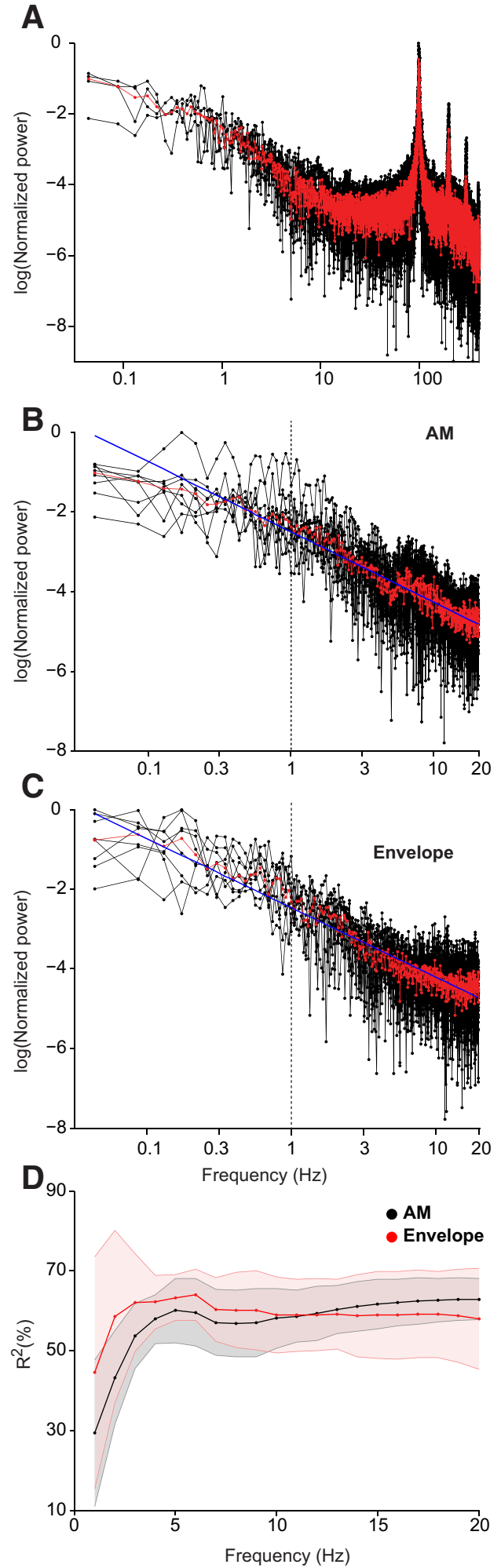

Figure 9. Spectral properties of the amplitude modulations in pairs of swimming fish. $\boldsymbol{A}$, Log-log plot of the normalized power spectra of the $V_{\text {tdp }}$ (black) and their median (red); data are from four recording sequences obtained from the same pair of fish used as an example in Figure $7 B$. The $V_{\text {tdp }}$ has power both at low frequencies, around the difference frequency of the two EODs $(100 \mathrm{~Hz})$ and its harmonics. $\boldsymbol{B}$, Log-log plot of the normalized power spectra of the low-pass $V_{\text {tdp }}$ (black) and their median (red, data are from eight recording sequences in two pairs of fish). The blue line is the fit to the median AM power with slope $=-1.70$ (SE $=0.03$ ), indicating a power-law relationship with an exponent of $\beta=1.70$. C, Log-log plot of the normalized power spectra of the envelopes (black) and their median (red, data are from eight recording sequences
$-1.83 ; \mathrm{SE}=0.25)$ was not significantly different from that fit to the full frequency range (Fig. 10AIII, red line, slope $=-1.89$; $\left.\mathrm{SE}=0.06 ; P_{\mathrm{ANCOVA}}=0.86\right)$, indicating a consistent power-law relationship for low and high frequencies.

Low-frequency drifts in transdermal potential were also observed during a recording sequence in which the fish swam slowly very close to a tank wall (Fig. $10 B I, B I I$, average swimming speed $=0.26 \mathrm{~cm} / \mathrm{s}$ ). Similar to the previous example, the power spectrum of this sequence could be well fit with a line over the full frequency range (Fig. 10BIII, red line, $R^{2}=69 \%$ ) and for frequencies smaller than $1 \mathrm{~Hz}$ (Fig. $10 B I I I$, blue line, $R^{2}=53 \%$ ), and the slope of the fit to frequencies smaller than $1 \mathrm{~Hz}$ (slope $=$ -1.63 ; SE $=0.34$ ) was not significantly different than that fit to the full frequency range $\left(\right.$ slope $=-2.1 ; \mathrm{SE}=0.06 ; P_{\mathrm{ANCOVA}}=$ 0.21 ). Conversely, during a recording sequence (from a different fish) in which the fish was hovering in place, making very small periodic position changes (Fig. 10CI, average swimming speed $=$ $0.05 \mathrm{~cm} / \mathrm{s}$ ), the $V_{\text {tdp }}$ showed only small periodic low-frequency fluctuations (Fig. 10CII). This recording sequence belonged to the second group (swimming speed $<0.25 \mathrm{~cm} / \mathrm{s}$ ), as described above, and its power spectrum flattened for large frequencies (Fig. 10CII). The power spectrum could be well fit with a line for the upper-bound frequency of $7 \mathrm{~Hz}$ (Fig. 10CIII, red line, slope = $\left.-1.83 ; \mathrm{SE}=0.12 ; R^{2}=63 \%\right)$. However, it could not be well fit with a line for frequencies smaller than $1 \mathrm{~Hz}$ due to the presence of peaks in the spectrum corresponding to the $V_{\text {tdp }}$ fluctuation frequency around $0.2 \mathrm{~Hz}$.

Finally, during a recording sequence in which the fish only made faster scanning movements near the tank walls (Fig. 10DI, average swimming speed $=1.16 \mathrm{~cm} / \mathrm{s}$ ), the $V_{\text {tdp }}$ fluctuations occurred at a relatively faster rate (Fig. 10DII). The power spectrum of this sequence could be well fit with a line over the full frequency range (Fig. 10DIII, red line, slope $=-2.14 ; \mathrm{SE}=0.06 ; R^{2}=$ $71 \%)$, however, the linear fit overestimated the power at lower frequencies (Fig. 10DIII) and the spectrum could not be fit as well for frequencies smaller than $1 \mathrm{~Hz}\left(R^{2}=16 \%\right)$, indicating the lack of a power-law relationship for low frequencies due to the absence of very slow movements.

Based on the above analysis, we predict that for a recording sequence long enough to encompass all possible swimming patterns of single fish (or relative swimming patterns of pairs of fish), the power spectrum of $V_{\text {tdp }}$ (and/or envelope) would follow a power-law relationship with frequency across all frequencies (with an upper frequency bound imposed by the largest possible movement frequency).

Power-law scaling of the spectrum indicates that spectral scale invariance, that is, the ratio of the signal power at any two frequencies, depends only on the ratio of those two frequencies and not their absolute values. Given a power exponent $1<$ $\beta<2$, as found for low-frequency AMs and envelopes, it can be shown that the underlying time series can be approximated as fractional Brownian motion with long-range temporal anticorrelations (Mandelbrot and Van Ness, 1968; Eke et al., 2002). Long-range anti-correlations tend to stabilize the temporal variations in that a change in one direction (e.g., an

$\leftarrow$

in two pairs of fish). The blue line is the fit to the median envelope power with slope $=-1.73$ $(\mathrm{SE}=0.03)$, indicating a power-law relationship with an exponent of $\beta=1.73$. $D$, Mean and $S D$ of the goodness of the linear fit $\left(R^{2}\right)$ for the AM and envelope of the individual recording sequences. The $R^{2}$ values of the linear fits to the power spectra of AMs (black) and envelopes (red) for various upper bound frequencies $(1-20 \mathrm{~Hz})$ were not significantly different $\left(p_{\text {KWT }}>0.05\right)$. 
A

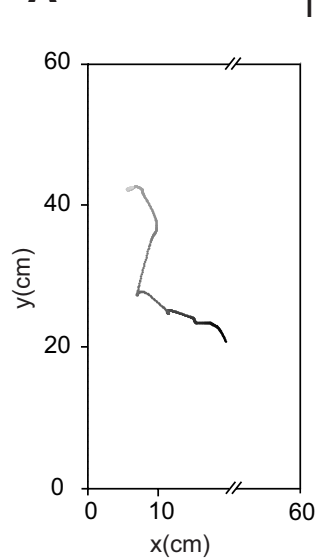

C

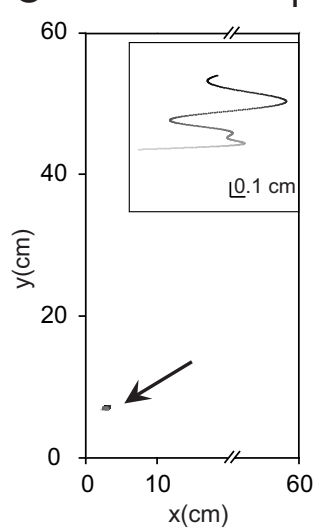

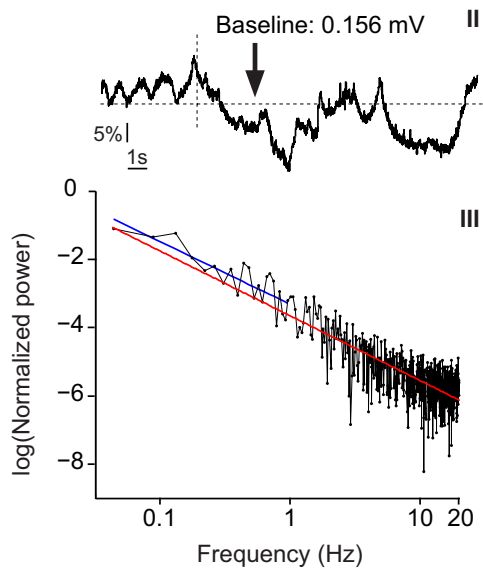

II

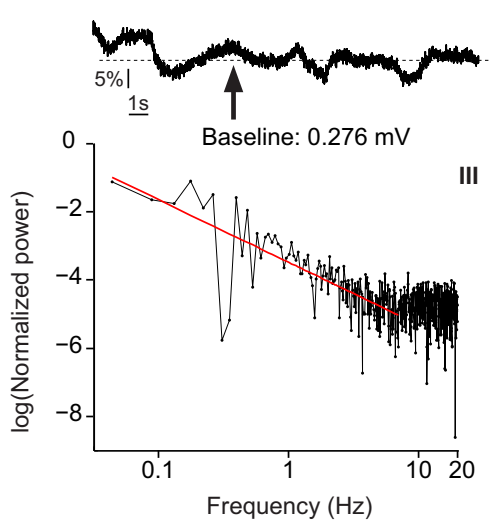

B
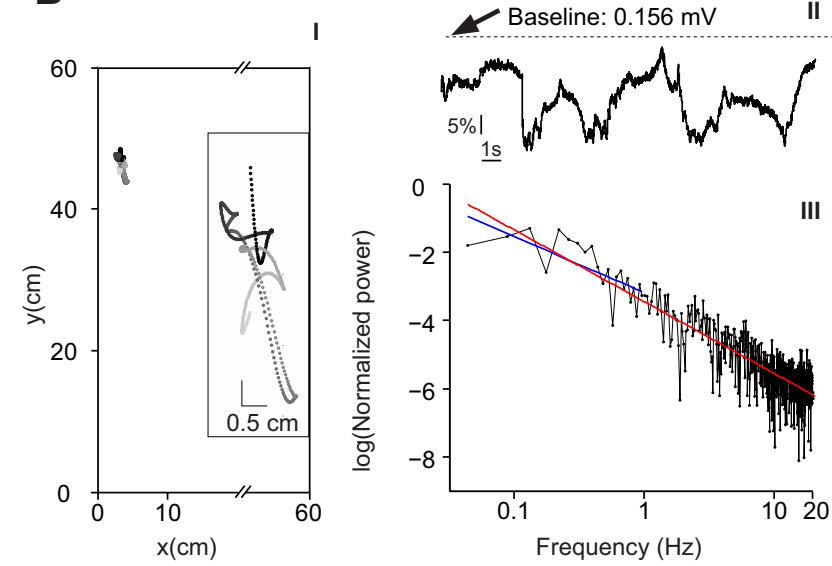

I

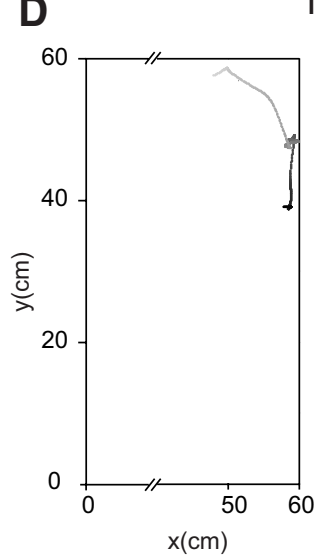

III

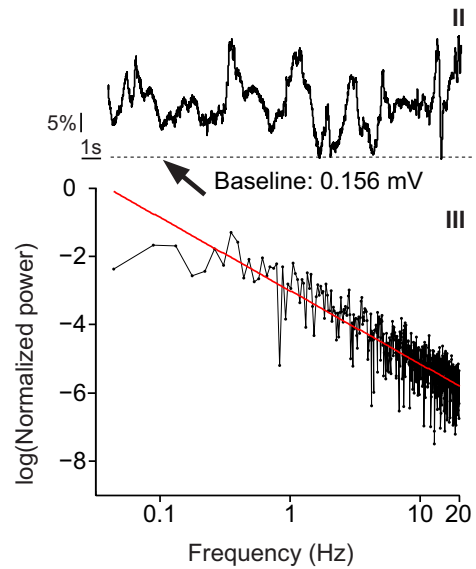

Figure 10. Example of the time course and power spectrum of $V_{\text {tdp }}$ evoked by distinct swimming patterns. Al, Fish location during the recording sequence from the top view. The position of the transmitter was tracked during the recording sequence and used to represent the location of the fish within the tank (lighter points represent fish position later in the sequence). The four side walls of the tank were located at $x=0, y=0, x=60$, and $y=60 \mathrm{~cm}$. The $x$-axis is shown broken to save space. The average swimming speed relative to walls closer than $10 \mathrm{~cm}$ (the wall at $x=0$ in this case) was $0.75 \mathrm{~cm} / \mathrm{s}$ in this example. $A$ III, The percent change in the $V_{\text {tdp }}$ acquired wirelessly relative to baseline TDP amplitude of $0.157 \mathrm{mV}$ marked by the horizontal dashed line ( $\Delta V_{\text {tdp }}$; see Materials and Methods). The electrode position in this example was ipsilateral to the wall at $x=0$. The vertical dashed line corresponds to the time point after which the electrode distance to the $x=0$ wall was smaller than $10 \mathrm{~cm}$. Approaching/receding from the tank wall $(x=0 \mathrm{~cm})$ caused slow drifts in $V_{\text {tdp }}$ in addition to faster fluctuations evoked by body movements. AIII, Power spectrum of $V_{\text {tdp }}$ could be well fit with a line in the log-log scale over the full frequency range ( $f<20 \mathrm{~Hz}$, red line; slope $=-1.89 ; \mathrm{SE}=0.06 ; R^{2}=66 \%$ ) and for frequencies smaller than $1 \mathrm{~Hz}$ (blue line; slope $=-1.83 ; \mathrm{SE}=0.25 ; R^{2}=72 \%$ ). $B \boldsymbol{B}$, Fish was swimming slowly back and forth near the tank wall at $x=0$ (average speed $=0.26 \mathrm{~cm} / \mathrm{s}$; the inset shows the movement pattern on an expanded scale). $\boldsymbol{B} I \mathbf{I}$, The percent change in the $V_{\text {tdp }}$ acquired wirelessly relative to baseline TDP amplitude marked by the horizontal dashed line ( $\Delta V_{\text {tdp }}$ same fish as in $\left.A\right)$. Slow swimming movements near the tank wall evoked low-frequency amplitude modulations in the $V_{\text {tdp }}$. The electrode position in this example was ipsilateral to the wall at $X=0$. BIII, Power spectrum of $V_{\text {tdp }}$ could be fit well with a line on the log-log scale for the full frequency range (red line; slope $=-2.1 ; \mathrm{SE}=0.06 ; R^{2}=69 \%$ ) and for frequencies smaller than $1 \mathrm{~Hz}$ (blue line; slope $=-1.63 ; \mathrm{SE}=0.34$; $R^{2}=53 \%$ ). CI, Fish was hovering in place in a corner of the tank (average speed $=0.05 \mathrm{~cm} / \mathrm{s}$; the inset shows the movement pattern on an expanded scale). $C$ II, The percent change in the $V_{\text {tdp }}$ acquired wirelessly, relative to the baseline TDP amplitude of $0.276 \mathrm{mV}$ marked by the horizontal dashed line. Slow swimming movements near the tank wall evoked low-frequency amplitude modulations in the $V_{\text {tdp }}$. The electrode position in this example was ipsilateral to the wall at $x=0$. CIII, The power spectrum of $V_{\text {tdp }}$ could be well fit with a line in the log-log scale up to $7 \mathrm{~Hz}$ (slope $=$ $\left.-1.83 ; \mathrm{SE}=0.12 ; R^{2}=63 \%\right)$, but not for frequencies smaller than $1 \mathrm{~Hz}\left(R^{2}=21 \%\right)$. For larger frequencies, the spectrum resembled that of the white noise. $D I$, Fish scanned the two tank walls located at $x=60 \mathrm{~cm}$ and $y=60 \mathrm{~cm}$, with an average speed of $1.16 \mathrm{~cm} / \mathrm{s}$, always remaining within $10 \mathrm{~cm}$ of either wall. DII, Percent change in the $V_{\text {tdp }}$ acquired wirelessly relative to baseline TDP amplitude marked by the horizontal dashed line $\left(\Delta V_{t d p}\right.$, same fish as in $\boldsymbol{A}$ and $\left.\boldsymbol{B}\right)$. The electrode position in this example was contralateral to the wall at $X=60$. $D I I I$, The power spectrum of the $V_{\text {tdp }}$ could be fit well with a line for the upper-bound frequency of $20 \mathrm{~Hz}$ (slope $=-2.14 ; \mathrm{SE}=0.064 ; R^{2}=71 \%$ ), but not for frequencies smaller than $1 \mathrm{~Hz}\left(R^{2}=16 \%\right.$ ).

increase in the amplitude) is more likely to be followed by one in the opposite direction (decrease in the amplitude). Assessing scale invariance and long-range temporal correlations in the time domain using detrended fluctuation analysis (Hardstone et al., 2012) led to similar results, as expected (data not shown), because the two methods are directly related (Heneghan and McDarby, 2000).

\section{Discussion}

Using a wireless transmitter system, we recorded the input to the electrosensory system of freely swimming weakly electric fish. This setup allowed us to characterize the statistics of electrosen- sory input taking into account the movement of the receptor array relative to the environment.

Body movements and displacement relative to nonconducting boundaries caused low-frequency AMs of $<20 \%$ of the baseline (Fig. 7A). We could predict the time course of the wirelessly measured AMs independently through the analysis of body movements and a computational model of the fish's electric organ (Fig. 4). Using this model, we show that specific body movements, such as side-to-side versus up-down tail movements, as well as approaches to non-conducting boundaries evoke distinct correlation patterns in the sensory input (Fig. 6). Such correlations contain information that may be used by the 
brain to guide behavior. There is now indeed evidence that weakly electric fish actively use their body movements to extract electrosensory information about the environment. During the search for prey, Apteronotus albifrons often tilts its body headdown, makes a rapid reversal in swimming direction once it detects prey, and shifts its body closer to it before finally capturing it (Nelson and MacIver, 1999; MacIver et al., 2001). Such swimming maneuvers result in a transient increase in local AM frequency, an increase in the size of the prey's image on the skin, and an increase in sensory volume (MacIver et al., 2010). The weakly electric fish Eigenmannia makes whole-body oscillations and tail bends while tracking the movement of an oscillating refuge (Stamper et al., 2012). Such movements induce changes in the spatiotemporal pattern of the input and electrosensory feedback and likely provide information to the brain about the relative location of the fish in its environment.

In the context of interaction with a conspecific, AMs could be as large as a $100 \%$ of the baseline (Fig. $7 C$ ) and had power at low frequencies and around the beat frequency (Figs. 9A). The degree of modulation around the beat frequency (envelope) was not a simple function of the distance between the two fish (Fig. 7D). In fact, a change in the relative orientation of the fish bodies during close interactions could significantly change the modulation depth, as predicted by an earlier modeling study (Kelly et al., 2008). Whether the fish actively alter their orientation relative to a conspecific as a means for electrical display behavior is an interesting subject for future studies. The spectral power of the $V_{\mathrm{tdp}}$ of a single swimming fish (Fig. 8A,B) and the low-frequency component of the $V_{\text {tdp }}$ of the fish during interactions with a conspecific as well its envelope (Fig. $9 B, C$ ) decrease with frequency according to a power law with the power exponent in the range of $1.5-1.8$, indicating the presence of spectral scale invariance and long-range temporal correlations in the natural electrosensory input.

The primary electrosensory afferents respond linearly to AMs smaller than 20\% (Gussin et al., 2007), which, interestingly, corresponds to the upper bound of the modulations we observe in a single swimming fish. Conversely, they show nonlinear behavior (saturation and rectification) for larger modulations (Bastian, 1981; Nelson et al., 1997; Gussin et al., 2007). In the context of interaction with conspecifics, therefore, the afferents are driven into their nonlinear coding range (Gussin et al., 2007), where they respond not only to the AMs, but also to their slowly varying envelopes (Savard et al., 2011). In the frequency domain, the afferents act as high-pass filters for $\mathrm{AM}$ frequencies in the range of $0.2-100 \mathrm{~Hz}$ (Hagiwara and Morita, 1963; Bastian, 1981; Xu et al., 1996; Nelson et al., 1997; Chacron et al., 2005), with the steepness of the gain-frequency curve increasing with frequency (Nelson et al., 1997). This property results in increased sensitivity to high-frequency AMs such as those encountered during interactions with conspecifics. For frequencies smaller than $20 \mathrm{~Hz}$, the slope of the afferent frequency response curve in the log-log domain is positive and smaller than 0.8 (calculated based on the proposed model of Nelson et al., 1997). Therefore, the afferents decorrelate, or "whiten," the sensory input with power-law statistics, although not completely in this case because the maximum positive slope of the filter's gain curve is smaller than the negative slope of the fit to the stimulus power spectrum.

Power-law scaling in space and time is an ubiquitous phenomenon in nature (Gisiger, 2001). Spectral power of images of natural scenes, the envelopes of natural sounds, and the temporal dynamics of pixel intensities across frames of videos taken from natural scenes follow a power-law relationship with frequency (Voss and Clarke, 1975; van Hateren, 1993; Ruderman and Bialek, 1994; Dong and Atick, 1995; Attias and Schreiner, 1997; Billock et al., 2001). Here we provide the critical result that power-law scaling of sensory input is maintained even when natural movements of the sensor array are taken into account, a factor that had not yet been included in measurements of sensory input statistics.

There is now much evidence that sensory systems exploit the power-law scaling and spatiotemporal correlation structures of natural scenes to efficiently encode sensory stimuli (Simoncelli and Olshausen, 2001). Remarkably, sensory adaptation in motion sensitive neurons of flies (Fairhall et al., 2001) and thalamic and cortical neurons of the rat vibrissa pathway (Lundstrom et al., 2008, 2010) act over a wide range of time scales depending on the time scale of the stimulus, making these neurons particularly suitable for encoding stimuli with power-law statistics. In the auditory system of cats, the modulation bandwidth of neurons in the central nucleus of the inferior colliculus scales with their characteristic modulation frequency, a property that opposes the power-law scaling in the envelope of natural sounds, and increases coding efficiency (Rodriguez et al., 2010). The envelope dynamics resulting from movements relative to a conspecific can be compared with the change in the intensity of an auditory stimulus arriving at the ears from a moving source. Specialized, well characterized circuitry within auditory nuclei including superior olive, lateral lemniscus, and inferior colliculus use interaural time difference or interaural intensity difference to localize sound (Joris et al., 2004; Pollak, 2012). It would be interesting to study the response characteristics of different elements of this circuitry using stimuli simulating a moving sound source with power-law statistics.

The electrosensory primary afferents provide input to distinct classes of pyramidal cells in the electrosensory lateral line (ELL) lobe of the hindbrain (Shumway, 1989; Krahe et al., 2008; Maler, 2009). The deep pyramidal cells respond linearly to stimuli and show little plasticity (Bastian et al., 2004), whereas the superficial ones receive extensive electrosensory and proprioceptive feedback (Sas and Maler, 1987; Bastian, 1995; Bastian and Nguyenkim, 2001) and respond nonlinearly to stimuli (Chacron, 2006). These cells are plastic and do not respond to periodic AMs caused by repetitive body movements due to the formation of a central "negative image" mediated by a cerebellar feedback pathway (Bastian, 1999). Based on our experiments, the movementevoked AMs could be non-repetitive and therefore may still drive those cells. It would be interesting to assess the spectral properties of the AMs in the context of specific electrolocation tasks such as prey capture because they might exhibit specific characteristics. For example, repetitive tail bends during the search for prey could cause the prey's image to stand out through disrupting the expected input correlation pattern. Enhanced responses to unpredictable sensory stimuli against ongoing predictable input have been reported in the context of interaction with a conspecific, in which the responses of ELL pyramidal cells to specific electrocommunication signals are enhanced in the context of ongoing predictable AMs at beat frequency (Marsat and Maler, 2012).

The information contained in the amplitude and envelope of sensory inputs is critical for sensory processing across sensory modalities and animal species. In weakly electric fish, the information contained in the AM and envelope of TDP is represented in the activity of cells in successive stages of electrosensory processing from electroreceptors (Savard et al., 2011) and ELL pyra- 
midal cells (Middleton et al., 2006) to cells in the midbrain (McGillivray et al., 2012). In the primary visual cortices of cats and monkeys, some neurons respond not only to sinusoidal gratings, but also to their time-varying contrast, which can be considered as a 2D envelope (Zhou and Baker, 1994; Mareschal and Baker, 1998). Neurons tuned to the envelope of sound stimuli are found at all stages of the vertebrate auditory system (Joris et al., 2004). In certain grasshopper species, a small set of auditory interneurons conveys information about the song envelope through their relative spike count and timing (Krahe et al., 2002). The processing of the stimulus and its envelope is commonly accomplished through parallel linear and nonlinear pathways and likely shares common neural mechanisms across sensory systems.

\section{References}

Assad C, Rasnow B, Stoddard PK (1999) Electric organ discharges and electric images during electrolocation. J Exp Biol 202:1185-1193. Medline

Attias H, Schreiner CE (1997) Temporal low-order statistics of natural sounds. Advances in Neural Information Processing Systems 9:27-33.

Babineau D, Longtin A, Lewis JE (2006) Modeling the electric field of weakly electric fish. J Exp Biol 209:3636-3651. CrossRef Medline

Barlow HB (1961) Possible principles underlying the transformation of sensory messages. In: Sensory communication (Rosenblith WA, ed.), pp 217-234. Cambridge: MIT.

Bastian J (1981) Electrolocation. 1. How the electroreceptors of Apteronotus albifrons code for moving objects and other electrical stimuli. J Comp Physiol A 144:465-479.

Bastian J (1995) Pyramidal-cell plasticity in weakly electric fish: a mechanism for attenuating responses to reafferent electrosensory inputs. J Comp Physiol A 176:63-78.

Bastian J (1999) Plasticity of feedback inputs in the apteronotid electrosensory system. J Exp Biol 202:1327-1337. Medline

Bastian J, Nguyenkim J (2001) Dendritic modulation of burst-like firing in sensory neurons. J Neurophysiol 85:10-22. Medline

Bastian J, Chacron MJ, Maler L (2004) Plastic and nonplastic pyramidal cells perform unique roles in a network capable of adaptive redundancy reduction. Neuron 41:767-779. CrossRef Medline

Billock VA, de Guzman GC, Scott Kelso JA (2001) Fractal time and 1/f spectra in dynamic images and human vision. Physica D: Nonlinear Phenomena 148:136-146.

Bullock TH, Hopkins CD, Fay RR, editors (2005) Electroreception. New York: Springer.

Carr CE, Maler L, Sas E (1982) Peripheral organization and central projections of the electrosensory nerves in gymnotiform fish. J Comp Neurol 211:139-153. CrossRef Medline

Chacron MJ (2006) Nonlinear information processing in a model sensory system. J Neurophysiol 95:2933-2946. CrossRef Medline

Chacron MJ, Maler L, Bastian J (2005) Electroreceptor neuron dynamics shape information transmission. Nat Neurosci 8:673-678. CrossRef Medline

Chen L, House JL, Krahe R, Nelson ME (2005) Modeling signal and background components of electrosensory scenes. J Comp Physiol A Neuroethol Sens Neural Behav Physiol 191:331-345. CrossRef Medline

Dong DW, Atick JJ (1995) Statistics of natural time-varying images. Network: Computation in Neural Systems 6:345-358. CrossRef

Egelhaaf M, Boeddeker N, Kern R, Kurtz R, Lindemann JP (2012) Spatial vision in insects is facilitated by shaping the dynamics of visual input through behavioral action. Front Neural Circuits 6:108. CrossRef Medline

Eke A, Herman P, Kocsis L, Kozak LR (2002) Fractal characterization of complexity in temporal physiological signals. Physiol Meas 23:R1-38. CrossRef Medline

Fairhall AL, Lewen GD, Bialek W, de Ruyter van Steveninck RR (2001) Efficiency and ambiguity in an adaptive neural code. Nature 412:787-792. CrossRef Medline

Fortune ES (2006) The decoding of electrosensory systems. Curr Opin Neurobiol 16:474-480. CrossRef Medline

Geisler WS (2008) Visual perception and the statistical properties of natural scenes. Annu Rev Psychol 59:167-192. CrossRef Medline
Gisiger T (2001) Scale invariance in biology: coincidence or footprint of a universal mechanism? Biol Rev Camb Philos Soc 76:161-209. CrossRef Medline

Gussin D, Benda J, Maler L (2007) Limits of linear rate coding of dynamic stimuli by electroreceptor afferents. J Neurophysiol 97:2917-2929. CrossRef Medline

Hagiwara S, Morita H (1963) Coding mechanisms of electroreceptor fibers in some electric fish. J Neurophysiol 26:551-567. Medline

Hardstone R, Poil SS, Schiavone G, Jansen R, Nikulin VV, Mansvelder HD, Linkenkaer-Hansen K (2012) Detrended fluctuation analysis: a scalefree view on neuronal oscillations. Front Physiol 3:450. CrossRef Medline

Harrison RR, Fotowat H, Chan R, Kier RJ, Olberg R, Leonardo A, Gabbiani F (2011) Wireless neural/EMG telemetry systems for small freely moving animals. IEEE Trans Biomed Circuits Syst 5:103-111. CrossRef Medline

Hedrick TL (2008) Software techniques for two- and three-dimensional kinematic mea surements of biological and biomimetic systems. Bioinspir Biomim 3:034001. CrossRef Medline

Heiligenberg W (1991) Neural nets in electric fish. Cambridge, MA: MIT.

Heneghan C, McDarby G (2000) Establishing the relation between detrended fluctuation analysis and power spectral density analysis for stochastic processes. Phys Rev E Stat Phys Plasmas Fluids Relat Interdiscip Topics 62:6103-6110. CrossRef Medline

Hofmann V, Sanguinetti-Scheck JI, Künzel S, Geurten B, Gómez-Sena L, Engelmann J (2013) Sensory flow shaped by active sensing: sensorimotor strategies in electric fish. J Exp Biol 216:2487-2500. CrossRef Medline Jackson JD (1975) Classical electrodynamics. New York: Wiley.

Joris PX, Schreiner CE, Rees A (2004) Neural processing of amplitudemodulated sounds. Physiol Rev 84:541-577. CrossRef Medline

Kayser C, Einhäuser W, König P (2003) Temporal correlations of orientations in natural scenes. Neurocomputing 52-54:117-123.

Kelly M, Babineau D, Longtin A, Lewis JE (2008) Electric field interactions in pairs of electric fish: modeling and mimicking naturalistic inputs. Biol Cybern 98:479-490. CrossRef Medline

Krahe R, Budinger E, Ronacher B (2002) Coding of a sexually dimorphic song feature by auditory interneurons of grasshoppers: the role of leading inhibition. J Comp Physiol A Neuroethol Sens Neural Behav Physiol 187: 977-985. CrossRef Medline

Krahe R, Bastian J, Chacron MJ (2008) Temporal processing across multiple topographic maps in the electrosensory system. J Neurophysiol 100:852867. CrossRef Medline

Kurtz R, Egelhaaf M (2003) Natural patterns of neural activity: how physiological mechanisms are orchestrated to cope with real life. Mol Neurobiol 27:13-32. CrossRef Medline

Lundstrom BN, Higgs MH, Spain WJ, Fairhall AL (2008) Fractional differentiation by neocortical pyramidal neurons. Nat Neurosci 11:1335-1342. CrossRef Medline

Lundstrom BN, Fairhall AL, Maravall M (2010) Multiple timescale encoding of slowly varying whisker stimulus envelope in cortical and thalamic neurons in vivo. J Neurosci 30:5071-5077. CrossRef Medline

MacIver MA, Patankar NA, Shirgaonkar AA (2010) Energy-information trade-offs between movement and sensing. PLoS Comput Biol 6:e1000769. CrossRef Medline

MacIver MA, Sharabash NM, Nelson ME (2001) Prey-capture behavior in gymnotid electric fish: Motion analysis and effects of water conductivity. J Exp Biol 204:543-547. Medline

Maler L (2009) Receptive field organization across multiple electrosensory maps. I. Columnar organization and estimation of receptive field size. J Comp Neurol 516:376-393. CrossRef Medline

Mandelbrot BB, Van Ness JW (1968) Fractional Brownian motions, fractional noises and applications. Society for Industrial and Applied Mathematics Reviews 10:422-437. CrossRef

Mareschal I, Baker CL Jr (1998) A cortical locus for the processing of contrast-defined contours. Nat Neurosci 1:150-154. CrossRef Medline

Marsat G, Maler L (2012) Preparing for the unpredictable: adaptive feedback enhances the response to unexpected communication signals. J Neurophysiol 107:1241-1246. CrossRef Medline

McGillivray P, Vonderschen K, Fortune ES, Chacron MJ (2012) Parallel coding of first- and second-order stimulus attributes by midbrain electrosensory neurons. J Neurosci 32:5510-5524. CrossRef Medline

Middleton JW, Longtin A, Benda J, Maler L (2006) The cellular basis for parallel neural transmission of a high-frequency stimulus and its low- 
frequency envelope. Proc Natl Acad Sci U S A 103:14596-14601. CrossRef Medline

Moore DS, McCabe GP (2006) Introduction to the practice of statistics, pp 637-667. New York: Freeman.

Nelson ME, MacIver MA (1999) Prey capture in the weakly electric fish Apteronotus albifrons: sensory acquisition strategies and electrosensory consequences. J Exp Biol 202:1195-1203. Medline

Nelson ME, Xu Z, Payne JR (1997) Characterization and modeling of P-type electrosen sory afferent responses to amplitude modulations in a wavetype electric fish. J Comp Physiol A 181:532-544. CrossRef Medline

Pollak GD (2012) Circuits for processing dynamic interaural intensity disparities in the inferior colliculus. Hear Res 288:47-57. CrossRef Medline

Rasnow B, Bower J (1996) The electric organ discharges of the gymnotiform fishes. 1. Apteronotus leptorhynchus. J Comp Physiol A 178:383-396.

Rasnow B, Assad C, Bower J (1993) Phase and amplitude maps of the electric or gan discharge of the weakly electric fish, Apteronotus leptorhynchus. J Comp Physiol A 172:481-491. CrossRef

Rodriguez FA, Chen C, Read HL, Escabí MA (2010) Neural modulation tuning characteristics scale to eficiently encode natural sound statistics. J Neurosci 30:15969-15980. CrossRef Medline

Ruderman DL, Bialek W (1994) Statistics of natural images: scaling in the woods. Phys Rev Lett 73:814-817. CrossRef Medline

Sas E, Maler L (1987) The organization of afferent input to the caudal lobe of the cerebellum of the gymnotid fish Apteronotus leptorhynchus. Anat Embryol 177:55-79. CrossRef Medline

Savard M, Krahe R, Chacron MJ (2011) Neural heterogeneities influence envelope and temporal coding at the sensory periphery. Neuroscience 172:270-284. CrossRef Medline

Schroeder CE, Wilson DA, Radman T, Scharfman H, Lakatos P (2010) Dynamics of active sensing and perceptual selection. Curr Opin Neurobiol 20:172-176. CrossRef Medline

Shumway CA (1989) Multiple electrosensory maps in the medulla of weakly electric gymnotiform fish. 1. Physiological differences. J Neurosci 9:4388-4399. Medline

Simoncelli EP, Olshausen BA (2001) Natural image statistics and neural representation. Annu Rev Neurosci 24:1193-1216. CrossRef Medline

Stamper SA, Roth E, Cowan NJ, Fortune ES (2012) Active sensing via movement shapes spatiotemporal patterns of sensory feedback. J Exp Biol 215: 1567-1574. CrossRef Medline

Van Hateren JH (1993) Spatiotemporal contrast sensitivity of early vision. Vision Res 33:257-267. CrossRef Medline

Voss RF, Clarke J (1975) 1/f noise in speech and music. Nature 258:317-318. CrossRef

Xu Z, Payne JR, Nelson ME (1996) Logarithmic time course of sensory adaptation in electrosensory afferent nerve fibers in a weakly electric fish. J Neurophysiol 76:2020-2032. Medline

Yu N, Hupé G, Garfinkle C, Lewis JE, Longtin A (2012) Coding conspecific identity and motion in the electric sense. PLoS Comput Biol 8:e1002564. CrossRef Medline

Zeimer RE, Tranter WH, Fannin DR (1990) Signals and systems: continuous and discrete. New Jersey: Prentice Hall.

Zhou YX, Baker CL Jr (1994) Envelope-responsive neurons in areas 17 and 18 of cat. J Neurophysiol 72:2134-2150. Medline 